\title{
Caractérisation au laboratoire de la sensibilité au retrait- gonflement des sols argileux
}

M. AUDIGUIER

Z. GEREMEW

S. LARIB|

R. COJEAN

Centre de géosiences, École des mines de Paris,

Bâtiment IFL, UMLV,

Cité Descartes

77454 Marne-la-Vallée

cedex 2

martine.audiguier@ensmp.fr zemenu.geremew@ensmp.fr

saoussen.laribi@ensmp.fr

roger.cojean@ensmp. fr
La présence de plusieur's formations argileuses affleurantes ou sub-affleurantes en région parisienne classe cette région parmi les plus valnérables à la sécheresse géotechnique. Deux formations sont plus particulièrement concernées : les Argiles vertes de Romainville et les Marnes bleues d'Argenteuil. Elles sont responsables du plus grand nombre de sinistres enregistrés sur le bâti depuis la loi du 13 juillet 1982 qui reconnaît la sécheresse géotechnique comme catastrophe naturelle. Une diminution anormale du tawx d'humidité du sol entraîne un phénomène de retrait, suivi lors des précipitations suivantes, d'un phénomène de gonflement dî à une réhumidification. C'est la conjugaison de ces deux facteurs (retrait et gonflement) qui est responsable des désordres observés sur le bâtti.

En prenant appui sur ces deux formations, une étude des facteurs de prédisposition au phénomène de retrait-gonflement des sols a été menée portant sur les caractéristiques minéralogiques, microstructurales, géotechniques et hydromécaniques. Cette étude met en évidence l'importance des minéraux argileux et plus particulièrement des smectites sur le phénomène de retrait-gonflement et sur les transformations microstructurales qui en découlent. Elle souligne aussi le róle que peuvent jouer d'autres minérawx (carbonates, pyrite) présents dans le sol sur l'évolution microstructurale du matériau naturel ou remanié, lors de l'hydiratation.

Les principaux paramètres géotechniques rendant compte de l'activité d'un sol argileux sont énoncés et quelques classifications basées sur ces paramètres sont données en exemple.

Enfin l'évolution de la microstructure de ces matériaux au cours des cycles séchage-humidification est analysée.

Mots-clés : sols argileux, sécheresse géotechnique, retrait-gonflement, minéralogie, microstructure, courbes de rétention, porosimétrie, microscope électronique à balayage. cycles séchage-humidification, gonflement libre.

\section{Laboratory characterization of clayed soils to shrinkage-swelling susceptibility}

The presence of expansive solls in some parts of the Paris area makes this area one of the most highly affected by droughtinduced geotechnical problems. Among these soils, the green clays of Romainville ("Argíes vertes de Rornainville") and the blue marls of Argenteuil ["Marnes bleues d'Argentevi]"] are responsible for the large amount of damage to buifdings as recorded since the swelling-shrinkage phenomenon was recognized as a "ratural catastrophe" according to the law" dated 13th July 1982. The shrinkage of the clayey soils while water is abrormatly evaporated during the summer season is followed by swelling while water infiltrates as a consequence of a long period of rainfall during the autumn or winter seasons. The combination of shrinkage and swelling results in damage observed on the buildings. 
Both formations were analysed in the laboratory by reference to shrinkage and swelling susceptibility. For this purpose, the mineralogy, the microstructure, the geotechnical and some hydromechanical parameters were characterized. This study highlights the importance of clay minerals, more particularly smectites, on the swelling-shrinkage behaviour of soils as well as on the resulting microstructural transformations. It underlises also the role of other minerals (carbonates, pyrite) on the microstructural evolution of the intact or remolded soils during the hydration process.

The principal geotechnical parameters for characterising the activity of a clay soll are stated and some classifications based on these parameters are given as exariples. Finally the microstructural evolution of these materials during cycles of wetting and drying is studied.

Key words: clay soil. geotechnical drought, shrinkage-swelling, mineralogy, microstructure, soil water retention curve, porosimetry scanning electron microscopy, wetting-drying cycles, free swelling.

1

\section{Introduction}

La sécheresse géotechnique est à l'origine de nombreux sinistres enregistrés en France sur les bâtiments et plus particulièrement sur les maisons individuelles fondées superficielement.

Elle résulte de l'évolution anomale du profil hydrique des sols vers des faibles teneurs en eau aprës des périodes de sécheresse climatique relativement longues ou survenant alors que l'état hydrique du sol n'est pas encore revenu à son profil d'équilibre. La sécheresse géotechnique se traduit par un phénomène de retrait des sols argileux suivi, à l'épisode de précipitations suivantes, par un phénomène de gonflement dû à une réhumidification.

La répétition des cycles de retrait-gonflement est responsable des désordres observés sur le bâti. En effet, à la fin de chaque cycle le sol ne revient pas totalement à l'état précédent, il subit des transformations microstructurales irréversibles qui sont responsables de déformations a l'échelle macroscopique. Ces dernières ont pour conséquence des mouvements du bâti compensés en partie d"wne saison à l'autre, mais qui globalement ont tendance à s'amplifier au cours du temps. Ainsi il peut s'écouler plusieurs mois, voire plusieurs saisons, avant que les premiers désordres n'apparaissent.

Un changement de l'état hydrique du sol se traduit gếnéralement par des varialions de volume dont l'origine provient de deux types de phénomènes :

- d'une part, des phénomènes physico-chimiques dus aux interactions entre l'eau interstitielte, la matrice poreuse et les particules argileuses ;

d'autre part, des phénomènes hydromécaniques découlant de l'histoire des contraintes subies par le matériau au cours des temps géologiques.

Ainsi à un état de contrainte totale donné, un changemenl dans l'environnement hydrique d"un sol conduit a une modification de la pression interstitielle ou de la succion qui, dans un sul saturé, est directement liée à une variation de l'état de contrainte effective par la relation de Terzaghi. Cette variation de contrainte effective est responsable d'une variation de volume. Dès que le sol se désature, il est nécessaire de prendre en compte les effets combinés liés aux variations de deux variables de contraintes indépendantes : la contrainte nette (o $-u_{2}$ ) et la succion ( $\left.u_{-}-u_{w}\right)$. La variation de volume est obtenue par le biais de modèles de comportement des sols non saturés de type surface d'état : e $=\mathrm{F}\left(\mathrm{o}-\mathrm{u}_{\mathrm{a}}, \mathrm{u}\right.$ - u (Matyas eil Radhakrisna, 1968) ou élastoplastique (modèle BBM, Alonso et al., 1990).

LIle-de-France, constituée essentellement de formations d'âge tertiaire où aitement des formations calcaires, gypseuses et argileuses (Fig. 1) est une des régions les plus touchées avec lá région Midi-Pyrénées et la région Poitou-Charente.

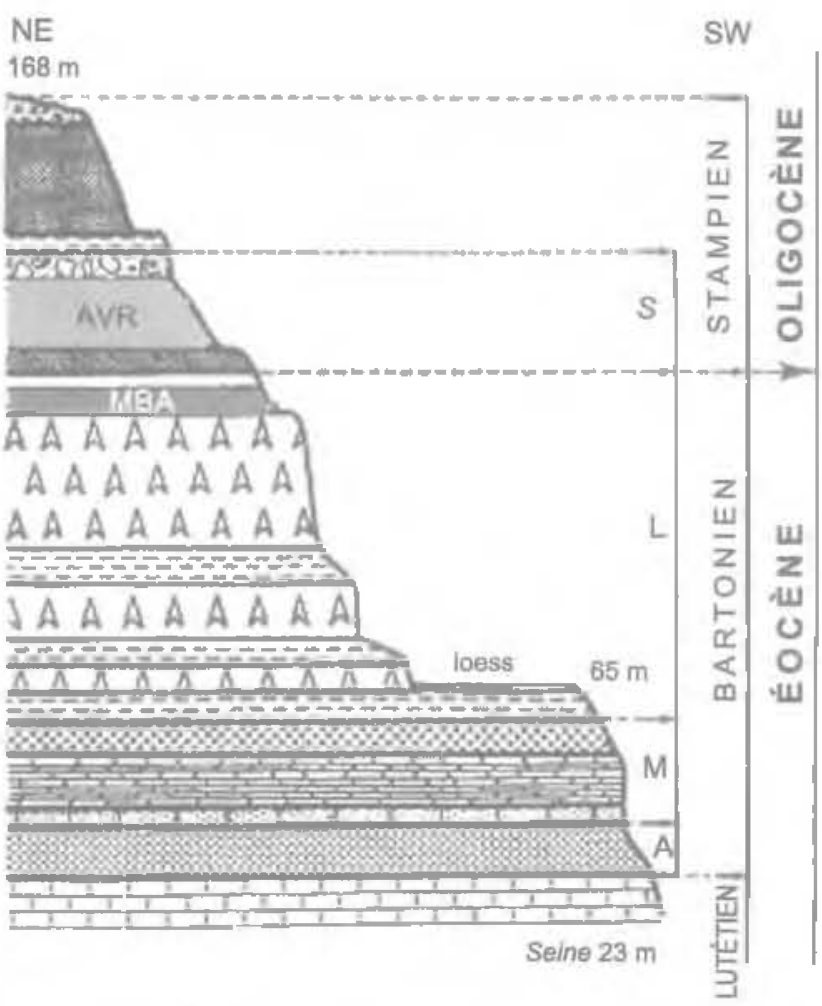

FG. 1 Coupe géologique de la butte de Cormeillesen-Parisis et localisation des Argiles vertes de Romainville (AVR) el des Marnes bleues d'Argenteuil (MBA).

Geological section of Comeilles-en-Parisis hill and location of Argiles vertes de Romainwille (AVR) and Marnes bleues d'Argenteuil (MBA). 
Parmi ces formations argileuses deux sont plus particulièrement sensibles au retrait-gonflement. Il s'agit des Marnes bleues d'Argenteuil (MBA) appartenant au Ludien supérieur, étage de l'Éocène supérieur et des Argiles vertes de Romainville (AVR) appartenant au Stampien inférieur, étage de l'Oligocène inférieur. Elles sont présentes sur l'ensemble de l'Ile-de-France et leur profondeur variable résulte du contexte géomorphologique. La vulnérabilité au retrait-gonflement de ces formations est d’autant plus grande qu'elles sont à l'affleurement, ou sous faible recouvrement, ou en situation de pente.

Les travaux présentés dans cet article s'appuient essentiellement sur l'Argile verte de Romainville. Les Marnes bleues d'Argentetil ne sont citées qu'à titre de comparaison pour souligner le rôle joué par la minéralogie dans le comportement des sols soumis à des sollicitations hydriques.

En prenant appui sur les résultats d’essais, les principales caractérisations au laboratoire de l'aptitude au retrait gonflement sont passées en revue et les conséquences de ce phénomène sur les modifications micros tructurales sont étudiées. Les aspects hydromécaniques sont abordés à travers l'étude microstructurale d'échantillons d'Argile verte de Romainville soumis à des cycles successifs de séchage - humidification.

2

\section{Minéralogie des sols argileux}

\section{1}

\section{Les minéraux argileux}

De par leurs propriétés cristallochimiques et électriques, les minéraux argileux jouent un rôle déterminant dans le processus de retrait-gonflement. Ils font partie de la famille des phyllosilicates et présentent donc une structure en feuillets. Le feullet élémentaire est constitué de deux couches

- la couche tétraédrique (couche T) formée par des tétraèdres dont les bases sont coplanaires et les sommets orientés dans une même direction. Ils forment un réseau plan à maille hexagonale. Les sommets sont occupés par des anions $\mathrm{O}^{2-}$ qui enserrent au centre un cation $\mathrm{Si}^{4+}$;

- la couche octaédrique (couche O) constituée par deux plans d'anions $\mathrm{O}^{2-}$ ou $\mathrm{OH}$ disposés en assemblage hexagonal compact. Le centre des octaèdres est occupé généralement par un cation $\mathrm{Al}^{3 *}$.

Les cations peuvent être substitués par des cations de valence moindre, $S 1^{4+}$ par $\mathrm{Al}^{3+} \mathrm{Ou} \mathrm{Fe}^{3+}$ ou Fe $\mathrm{F}^{2+} \ldots$; $\mathrm{Al}^{3-1}$ par $\mathrm{Fe}^{3 *}$ ou $\mathrm{Fe}^{2+}$ ou $\mathrm{Mg}^{2+}$... Les deux couches sont interdépendantes : les $\mathrm{O}^{2}$ des sommets des tétraèdres sont partagés avec un plan de la couche octaédrique.

L'assemblage de ces deux couches permet de définir plusieurs types de feuillets élémentaires qui constituent différentes familles de minéraux argileux.
Trois familles principales seront abordées ici : - la famille de la kaolinite (Fig. 2) est constituée d"une couche $T$ et d'une couche $O$ et ne présente que peu ou pas de substitution. L'épaisseur du feuillet élémentaire est de $7 \AA$.

Les particules argileuses se présentent sous forme de cristallites par association de plusieurs feuillets reliés entre eux par des liaisons hydrogènes ne laissant aucune possibilité de passage aux molécules d'eau (Tessier, 1984). Elles forment ainsi des plaquettes rigides de quelques centaines de nanomètres à quelques microns d'extension et de quelques dizaines cie nanomètres d'épaisseur ;

- la famille de l'illite (Fig.3) de type TOT est constituée d'une couche octaédrique entourée de deux couches tétraédriques. Le feuilet élémentaire a une épaisseur de $10 \AA$. Ce feuillet élémentaire présente des substitutions isomorphiques dens la couche I (Si par Al) et/ou dans la couche O (Al par Mg. Mg par Li. ...) créant ainsi un déficit de charge comblé par des cations $K^{*}$ qui ont exactement la dimension des cavités de surface du feuillet ou ils sont piégés et qui servent de lien entre les feuillets. Cette liaison par le cation $\mathrm{K}^{-}$ est si forte que les molécules d'eau ne peuvent accéder à l'espace interfoliaire. Les cations $K^{+}$sont non hydratables et non échangeables (Tessier, 1984). Les particuJes d'illite se présentent aussi, comme la kaolinite, sous forme de cristallites de quelques dizaines de feuillets ;

- la famille des smectites (Fig. 3) est de mểme configuration que la précédente mais présente un déficit de charge plus faible, comblé dans l'espace interfoliaire par des cations compensateurs hydratables tel que le sodium ( $\mathrm{Na}^{+}$) ou le calcium ( $\mathrm{Ca}^{+1}$ ). L'épaisseur des feuillets varie généralement entre 12 et $20 \AA$ selon la nature et l'hydratation du cation. La liaison entre les feuillets, de faible énergie, est assurée par les cations compensateurs. Les particules de smectite sont caractérisées par une grande extension (1 $1 \mathrm{~mm}$ ) par rapport à l'épaisseur, leur conférant un aspect rubané (Fig. 4).

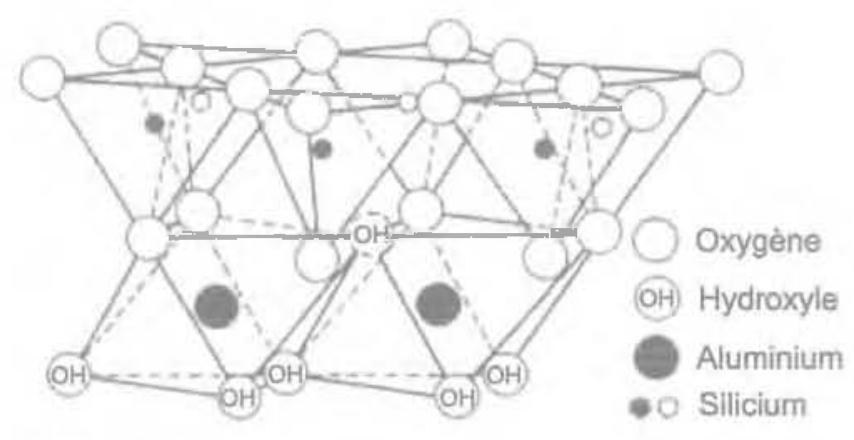

Fig. 2 Schéma structural de la kaolinite (d'après Mitchell et Soga, 2005).

Dlagram of the kaclinite structure (after Mitcheil and Soga, 2005)

A ces trois familles principales s'ajoute celle des interstratifiés qui regroupe les minéraux argileux formés par une succession de feuillets élémentaires appartenant à deux familles d'argiles différentes. I existe deux types d'interstratifiés, les interstratifiés réguliers : alternés $(A B A B)$ ou ségrégés (AAABBB) et les interstratifiés irréguliers dont l'empilement des feuillets ne présente aucune périodicité. Les interstratifiés illite-smectite, de différents types, sont très fréquents dans les sols argileux présentant une aptitude au retrait-gonflement. 


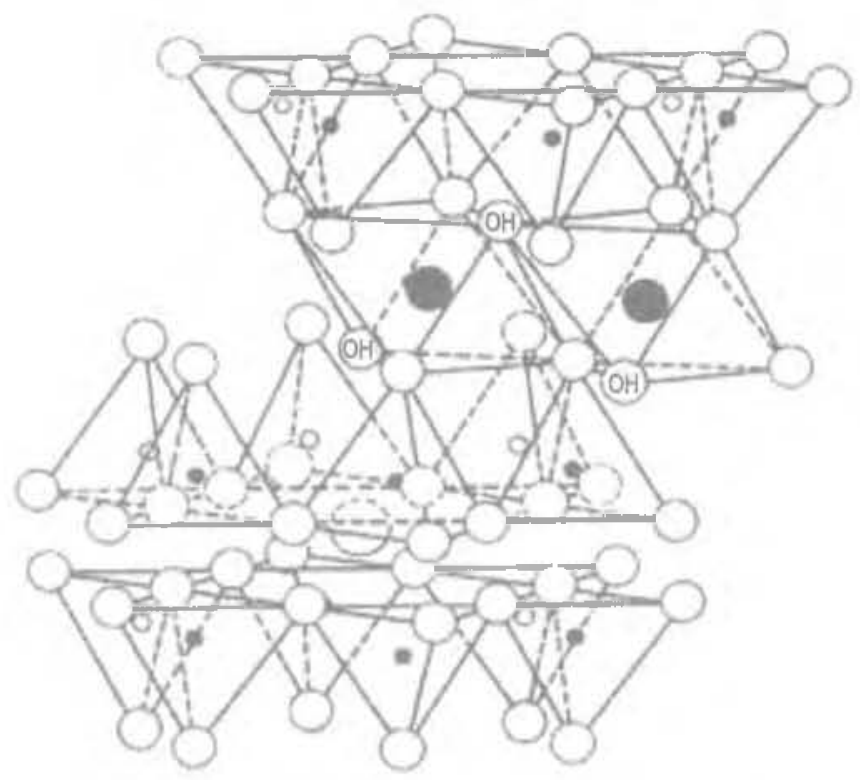

Oxygène @Hydroxyle Aluminium Potassium

o et • Silicium (1 sur 4 est remplacé par 1 Aluminium)

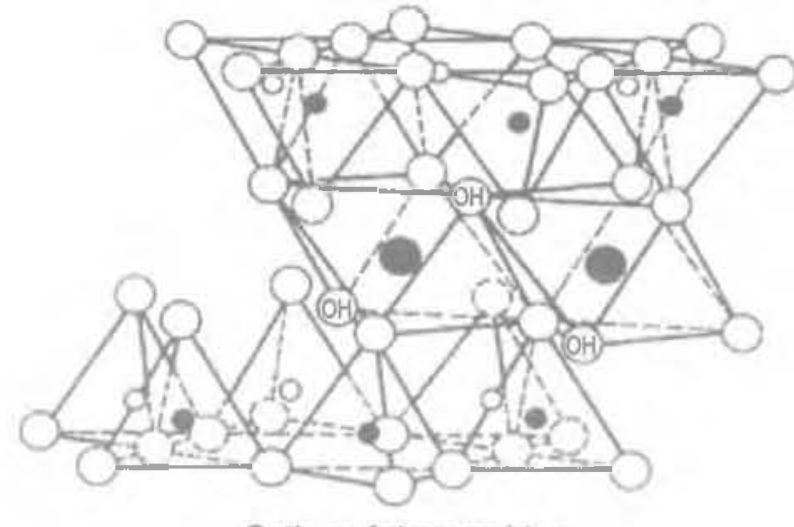

Cations échangeables

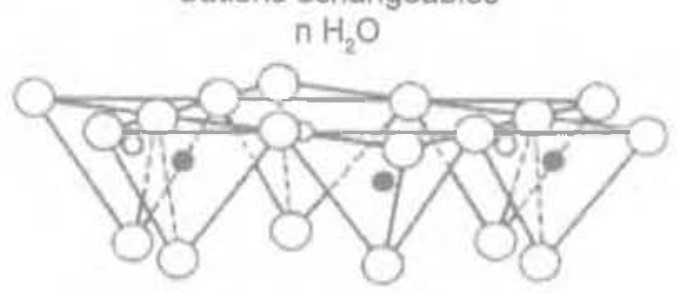

Oxygène Hydroxyle Aluminium, Fer, Magnésium

et $\bullet$ Silicium, Aluminium

Fa. 3 Schémas structural de l'illite (gauche) et des smectites (diroite). (d'après Mitchell et Soga, 2005). Diagrams of the ilite structure (lieft) and smectite structure(right), (after Mitchell and Soga, 2005).

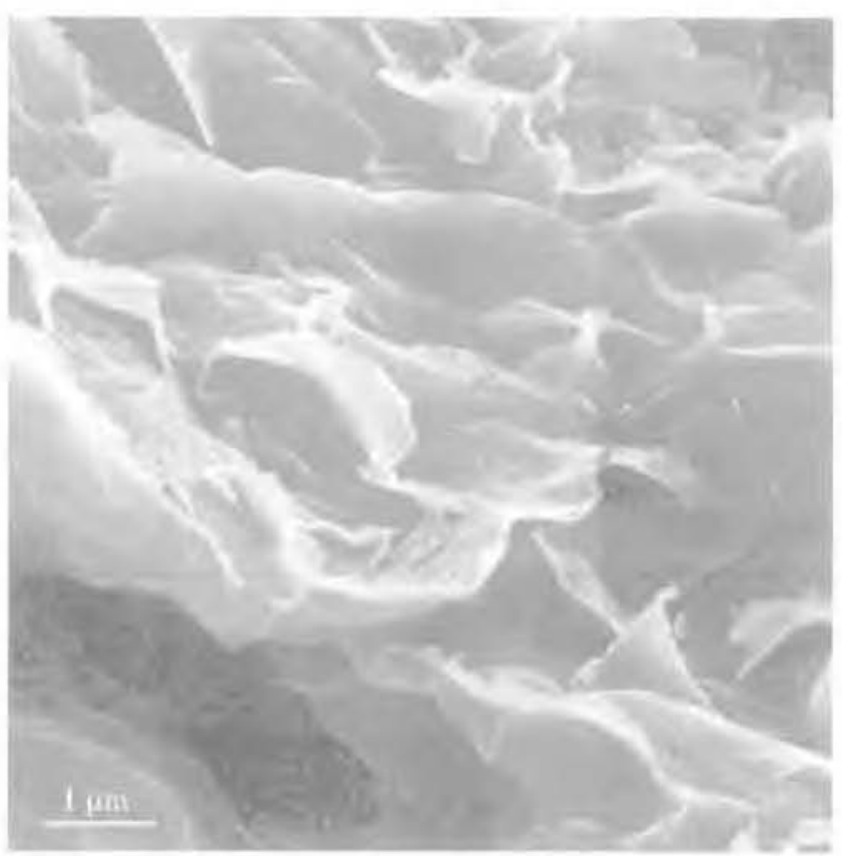

Fi. 4 Image en microscopie à balayage de smectite (d'après Tovey 1971, dans Mitchell J. K. 1976).

SEM picture of smectite (after Tovey 1971. in Mitchell I.K 1976).
Les minéraux argileux contenus dans les Argiles vertes de Romainvilie et les Marnes bleues d'Argenteuil sont représentés par de l'ilite, de la kaolinite, des smectites et des interstratifiés illite-smectite.

\section{9}

\section{Relation eau-particules argileuses}

Comme il a été souligné dans le paragraphe précédent le processus d"hydratation affecte essentiellement les smectites. 11 comporte différentes échelles :

- le déficit de charge des particules argileuses leur confère un pouvoir d'adsorption de dipôles d"eau sur leur surface externe. Dans le cas des smectites, à la surface externe s'ajoute la surface interne lou interfoliaire) qui, étant donné la morphologie des particules, peut être considérable. Ceci est mis en évidence par les travaux de Salyouri ef al (2000, 2004) synthétisés dans le graphe de la figure 5 (Delage, 2007). L'étude de la diffraction $\mathrm{RX}$ aux petits angles de l'hydratation d'une smectite MX80 compactée, dont les résultats sont analysẻs à partir du nodèle probabiliste de Pons [Pons et al., 1981), montre qu'au fur et à mesure de l'hydratation (et de la diminution de la succion à lacuelle est soumise l'échantillon), la distance entre les feuillets élémentair'es augmente par adsorption progressive de couches d'eau. Ainsi pour une succion inférieure à $50 \mathrm{MPa}$ l'hydratation intraparticulaire passe de une a deux couches d'eau, à partir de $7 \mathrm{MPa}$ elle est de trois couches pour atteindre quatre couches dans le cas d'une suc- 
cion quasi nulle. Dans le même temps les particules se divisent et leur épajsseur passe de plusieurs centaines de feuillets à une dizaine, augmentant ainsi de façon considérable l'espace interparticulaire accessible à l'eau ;

- à l'échelle de l'agrégat, prend place l'eau adsorbée par condensation capillaire aux points de contact entre les particules ou les agrégats (Prost, 1990; Van Damme, 2002).

Certains auteurs (Durand, 1995; Qi, 1996) parlent. d'eau interne à l'échelle de la particule et d'eau externe à l'échelle de l'agrégat et entre les agrégats. La proportion d'eau interne et externe dépend de la nature de l'argile, des cations échangeables, de la température et de la salinité de l'électrolyte. Ainsi la présence de $\mathrm{Na}^{*}$ et $\mathrm{Mg}^{*}$ favorise l'eau inlerne. A l'opposé les cations $\mathrm{Ca}^{2+}$ et $\mathrm{K}^{*}$ favorisent l'eau externe. De mêmen de fortes températures ou salinités sont défavorables à l'adsorption d'eau interne (Turki, 1987).

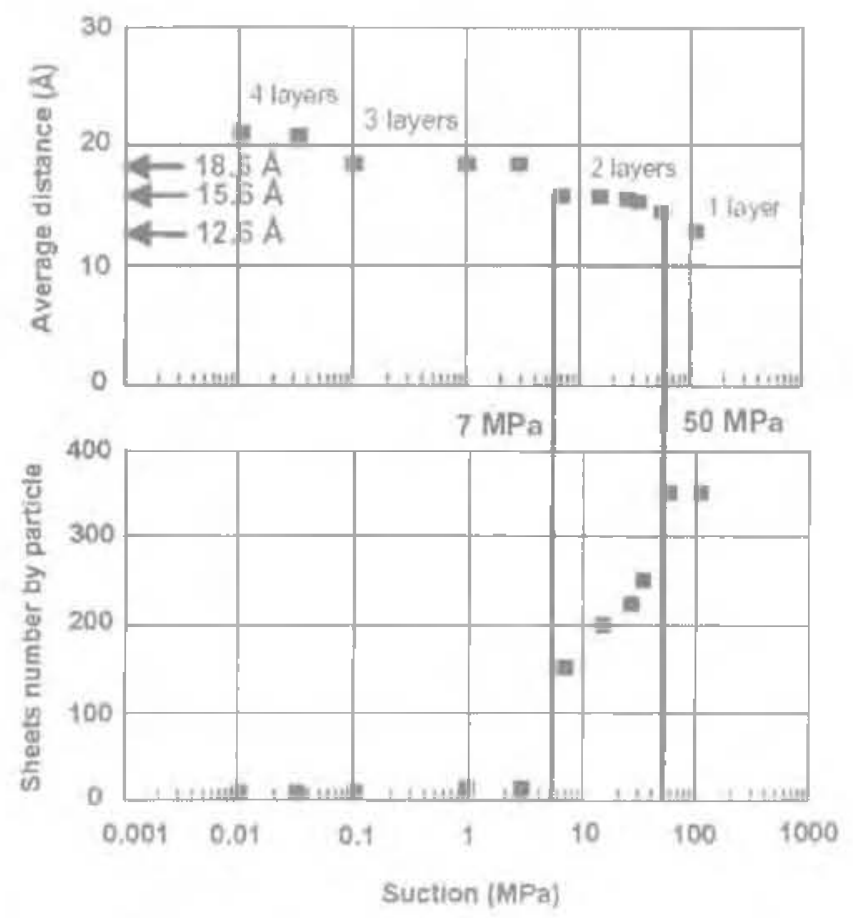

Fig.s Effet d'une diminution de la succion (par hydratation) sur l'argile MX80 augmentation du nombre de couches d'eau adsorbée, diminution du nombre de feuillets par particule (d'après Saiyouri et al., 2000, 2004, dans Delage, 2007).

Effect of a suction decrease (due to hydration) on the MX80 particles: increase in the nutiber of adsorbed water layers, clecrease in the number of stacked layers per particle (after Sayour et al'. 2000, 2004, in Dolage, 2007).

\section{Minéraux non argileux}

Les sols argíleux sont composés, outre les argiles, d'autres minéraux en proportions variables qui son susceptibles de jouer également un rôle dans le processus de retrait-gonflement. Ainsi, la plupart des sols argileux de la région parisienne contiennent des carbonates sous forme de calcite et de dolomite. Afin d'il-

lustrer le rôle joué par ces minéraux dans le processus de retrait-gonflement, sont présentés ici des résultats d'essais réalisés sur les deux formations évoquées en introduction : l'Argile verte de Romainvilie (AVR) et la Marne bleue d'Argenteuil (MBA) (Tableau I).

ta.ueall Caractéristiques minéralogiques, géotechniques et mécaniques de l'Argile verte de Romainville et de la Marne bleue d'Argenteuil.

Mineralogical, geotechnical and mechanical characteristics of Argile verte de Ronainville and Marne bleue d'Argenteuil.

\begin{tabular}{|c|c|c|}
\hline Formations & $\begin{array}{l}\text { Argiles vertes } \\
\text { de Romainwile }\end{array}$ & $\begin{array}{l}\text { Marnes bleues } \\
\text { d'Argenteuil }\end{array}$ \\
\hline Argiles & K, L, Interstratifies U/S. & $K, I, S$ \\
\hline$\%$ Carbonates & $15-20$ & $30-60$ \\
\hline$\%<2 \mu m$ & $80-90$ & $80-90$ \\
\hline $\begin{array}{l}\text { Linite de liquidité } \\
W_{1} \%\end{array}$ & 75 & 85 \\
\hline $\begin{array}{l}\text { Indice de } \\
\text { plasticite Ip } \%\end{array}$ & $30-35$ & $40-50$ \\
\hline $\begin{array}{l}\text { Indice de retrait } \\
\text { Ir \% }\end{array}$ & $55-60$ & $65-75$ \\
\hline $\begin{array}{l}\text { Gonflement libre } \\
\text { etat naturel \% }\end{array}$ & $15-25$ & 4 \\
\hline $\begin{array}{l}\text { Gonflemert libre } \\
\text { etat remanié \% }\end{array}$ & 330 & 26 \\
\hline
\end{tabular}

Ces deux formations sont des matériaux fins dont le pourcentage de particules inférieures à 2 um est supérieur à $80 \%$. Leur composition minéralogique est voisine; outre les minéraux argileux évogués dans le paragraphe précédent, les autres minéraux présents sont essentiellement du quart? et des carbonates : calcite et dolomite en proportions variables. Ces carbonates sont plus représentés dans les Marnes bieues d'Argenteuil. Les limites d'Atterberg ( $w_{L}, I_{n}, I_{r}, \ldots$ ) les classent dans les matériaux à fort potentiel de gonflement (voir tableau I).

Des essais de gonflement libre ont été réalisés sur des échantillons intacts et des échantillons remaniés à la limite de liquidité et ramenés à la teneur en eau initiale. Dans cet essai, un échantillon est placé dans un moule oedométrique, sous une charge très faible (de l'ordre du poids du piston) en contact, par l'intermédiaire d'une pierre poreuse, avec de l'eau à la pression atmosphérique. Le taux de gonflement libre, rapport entre la déformation maximale de l'éprouvette et la hauteur initiale, est mesuré.

Les résultats de ces essais montrent que les gonflements libres des échantillons de AVR intacts et remaniés ne varient que de quelques pourcents avec cependant un résultat légèrement supérieur dans le cas de l'échantillon remanié. Pour les échantillons de MBA le gonflement de l'échantillon remanié est six fois plus grand que celui de l'échantillon intact. Ce résultat peut certainement être attribué à la présence des carbonates dont le pourcentage est plus important dans les échantillons de MBA que dans les échantillons de AVR. Ces minéraux contribuent à l'édification d'un squelette rigide qui s'oppose au gonflement, mais qui est néanmoins fragile puisque détruit par le remantement.

La pyrite est présente de façon accessoire, généralement concentrée en amas ou lit (Fig. 6). La pyrite s'oxyde en présence d'oxygène pour donner des sulfates qui par association avec des lons $\mathrm{Ca}^{++}$forment de l'anhydrite $\left(\mathrm{CaSO}_{4}\right)$. En présence d'eau l'anhydrite se 
transforme en gyose ( $\mathrm{CaSO}, 2 \mathrm{H}_{3} \mathrm{O}$ ). Ces transformations sont responsables de pressions de gonflement et de taux de gonflement importants, phénomènes connus et redoutés des géotechniciens car souvent à l'origine de désordres dans les terrassements.

\section{3}

\section{Microstructure des sols argileux}

\section{Définitions}

Dans les sols argileux, les particules argileuses et les grains minéraux non argileux, en proportions variables, forment un arrangement structural illustré par la représentation schématique de Colkins et Mc Gown (1974) (Fig. 7). Dans les sols de faible derisité, les minéraux se rassemblent pour donner une structure en agrégats plus ou moins réguliers, formés par des argiles et/ou des grains minéraux. Ces agrégats sont reliés par des ponts argileux. Dans les sols plus denses, la notion de structure en agrégats n'est pius applicable car les agrégats ne sont plus distincts les uns des autres, on parle alors de structure matricielle a dominante argileuse ou granulaire.

La plupart des auteurs, sous des terminologies différentes (Yong, 1999; Gens et Alonso, 1992), s'accordent à définir un schéma d'organisation des sols gonflants en trois niveaux structuraux gui jouent un rôle déterminant dans le processus de retrait-gonflement. (Fig. 8) : - infrastructure = particule argileuse ;

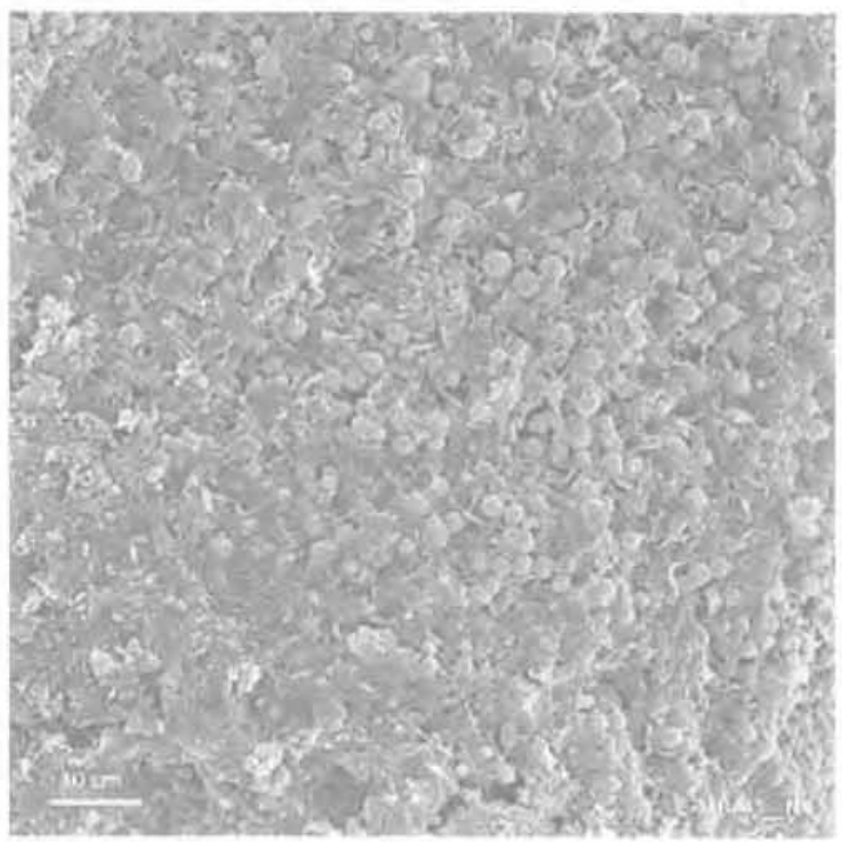

flg. 6 Image prise au MEB dramas de grains de pyrite dars la Marne bleue d'Argenteuil. SEM picture of pyrite lers in marne bleue d'Aryenteuil.

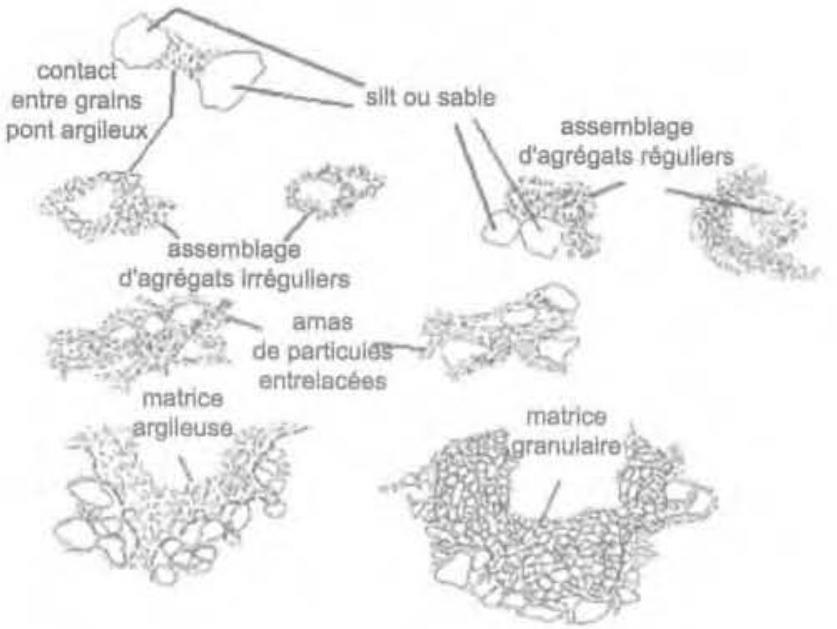

7g. 7 Représentations schématiques d'assemblages de particules argileuses et de grains non argilewx (d'après Collins et Mc Gown, 1974).

Schematic representations of particle aggregations of clay particles and no clayey grains lafter Collins and Mc Gown. 1974).

- microstructure = agrégats (clusters en anglais) formés par les particules argileuses et les autres composants du sol;

- macrostructure $=$ assemblage macroscopique des agrégats formant les « peds .

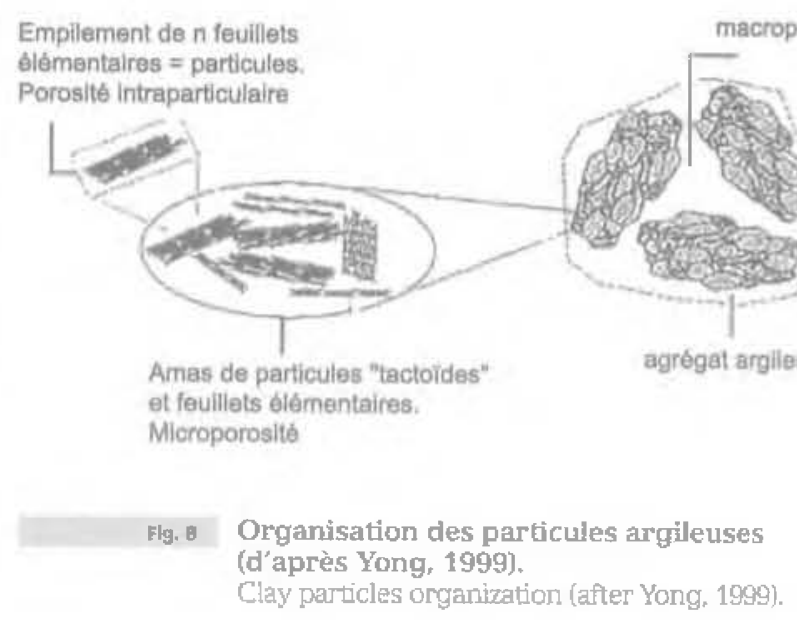

Cet arrangement stuctural détermine la forme et la taille du réseau poreux associé. A partir d'un sol contenant essentiellement des minéraux argileux de type smectitique, Touret (1990) a défini trois classes ou familles de pores (Fig. 9) qui sont aussi applicables à des sols contenant une phase granulare:

- la porosité interfoliaire et lenticulaire ou intraparticulaire :

- la porosité interparticulaire ou intra agrégat :

- la porosité interagrégat. 


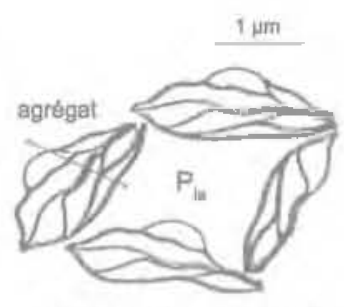

Représentation schématique d'un arrangement d'agrégats. $P_{\text {sin }}$; pore Interagrégats

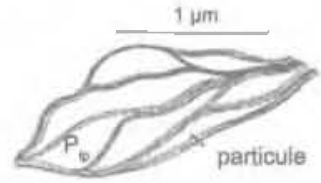

Dótall d'un agrégat constitué par assoclation de particules. $P_{\text {s. }}$ : pore interparticulaire

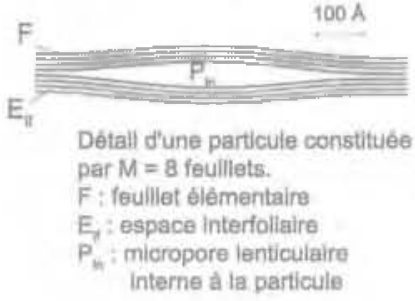

7ig. Représentation schématique des différents types de pores dans des smectites (d'après Touret, 1990).

Schematic representation of pore families in smectite (after Touret, 1990).

La taille moyenne des différentes classes varie suivant les auteurs. Cependant, en s'appuyant sur une synthèse de résultats bibliographiques, Al-Mukhtar et al. (1996), Robinet et al. (1996) donnent les valeurs moyennes suivantes : 1 à 2,5 nm pour la porosité intraparticulaire, 2,5 à 150-200 nm pour la porosité interparticulaire et supérieure à 150-200 nm pour la porosité interagrégat.

\section{$3 . ?$}

\section{Exemples}

A titre d'exemple, les caractérisations structurales de l'état naturel de l'Argile verte de Romainville (AVR, $w=25 \%$ ) et de la Marne bleue d'Argenteuil (MBA, $w=30 \%$ s sont présentées, ainsi que celles de l'Argile verte de Romainville sous deux configurations lithologiques différentes. Ces caractérisations sont, d'une part, qualitatives à partìr de l'observation au microscope électronique à balayage (MEB) et, d'autre part. quantitatives par la mesure de la distribution des diamètres d'accès de pores par porosimétrie au mercure. en se rétérant à la loi de Jurin eppliquẻe à un modèle de pores cylindriques (Fig. 10 et 11).
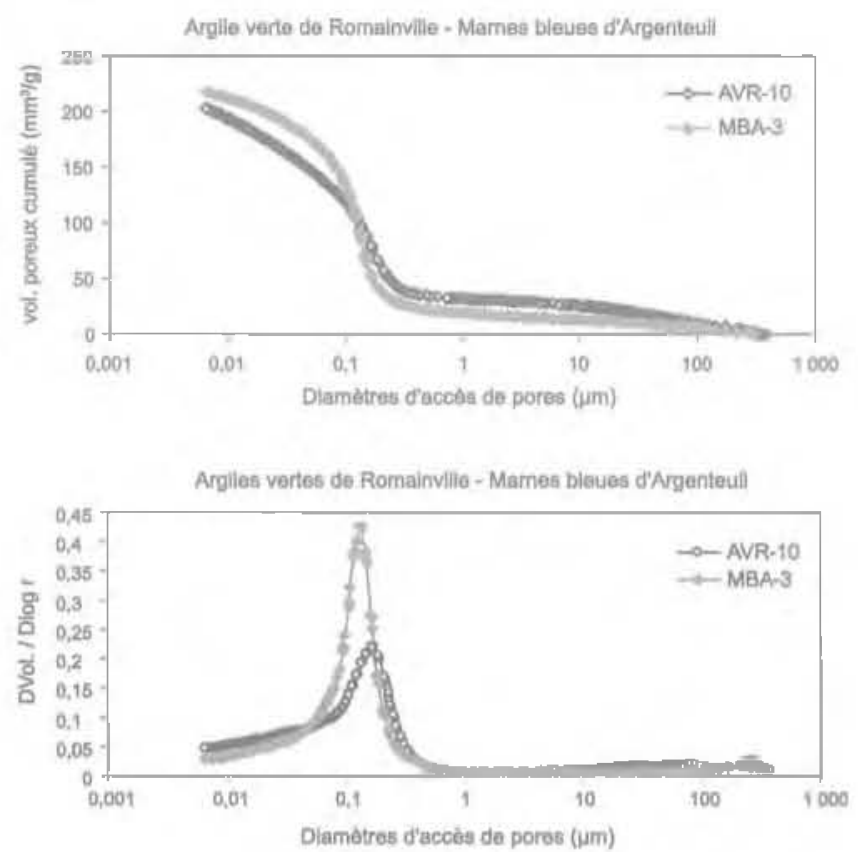

Fy. 10 Courbes cumulées et dérivées de distribution des diamètres de pores de I'Argile verte de Romainville et des Marnes bleues d'Argenteuil.

Cumulative and derived curves of pore size distribution of argile verte de Romainville and mames bleues d'Argenteuil.

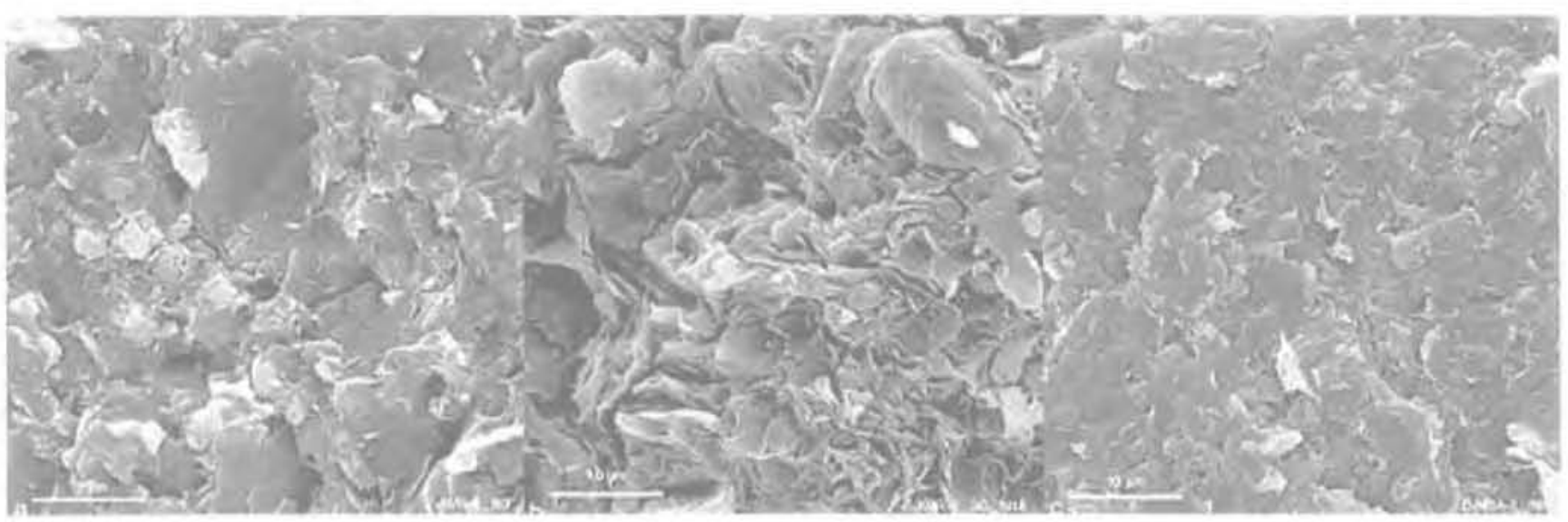

Fig. 11 Inages au MEB. a) Argile verte de Romainville : plan parallèle au litage, b) Argile verte de Romainville : plan perpendiculaire au litage, c) Marne bleue d'Argenteuil : plan parallèle au litage.

SEM micrograph. al Argile verte de Romainville : view parallel to the bedđing, b) Argile verte de Romainville : view perpendictiar to the bedding, c) Marne bleue d'Argenteuil : view parallel to the bedding. 
Les distributions obtenues sur les deux formations sont unimodales centrées entre 100 et $200 \mathrm{~nm}$. Elles présentent une queue de distribution vers les petits diamètres, dénotant une porosité interparticulaire et intraparticulaire non accessible par la porosimétrie au mercure. Ces courbes sont caractéristiques d"une structure matricielle argileuse (Audiguier et Delage, 1987) dans laquelle l'architecture en agrégats n'apparaît pas. L'observation des deux formations au MEB montre que la microstructure est compacte et qu'elle présente un arrangement préférentiel des particules argileuses parallèlement au litage.

La comparaison des courbes de distributions de l'Argile verte de Romainvile intacte $(w=25 \%)$ et sous forme de colluvions argileuses ( $w=32 \%$ ) (Fig. 12) met en évidence l'existence d'une famille vers les très faibles diamètres (porosités inter et intraparticulaire), dans le cas des colluvions. Cette famille dénote une déstructuration du matérlau qui favorise l'apparition de particules argileuses dispersées ou sous forme d'amas de quelques particules.

Afin de metre en évidence les changements de microstructure au cours du séchage ou de l'humidification, trois échantillons d'Argile verte de Romainville ont été étudiés à différents états hydriques :

- séché à l'étuve:

- naturel (teneur en eau $25 \%$ ), lyophilisé : la lyophilisation est une méthode de déshydratation qui consiste en une congélation rapide des échantillons dans de l'azote liquide amené à son point de congélation $\left(-210^{\circ} \mathrm{C}\right)$ par application du vide, suivi d'une phase de sublimation sous vide en plaçant les échantillons dans un lyophilisateur (Delage et Pellerin, 1984) :

- après gonflement libre (teneur en eau $39 \%$ ), Iyophilisé.
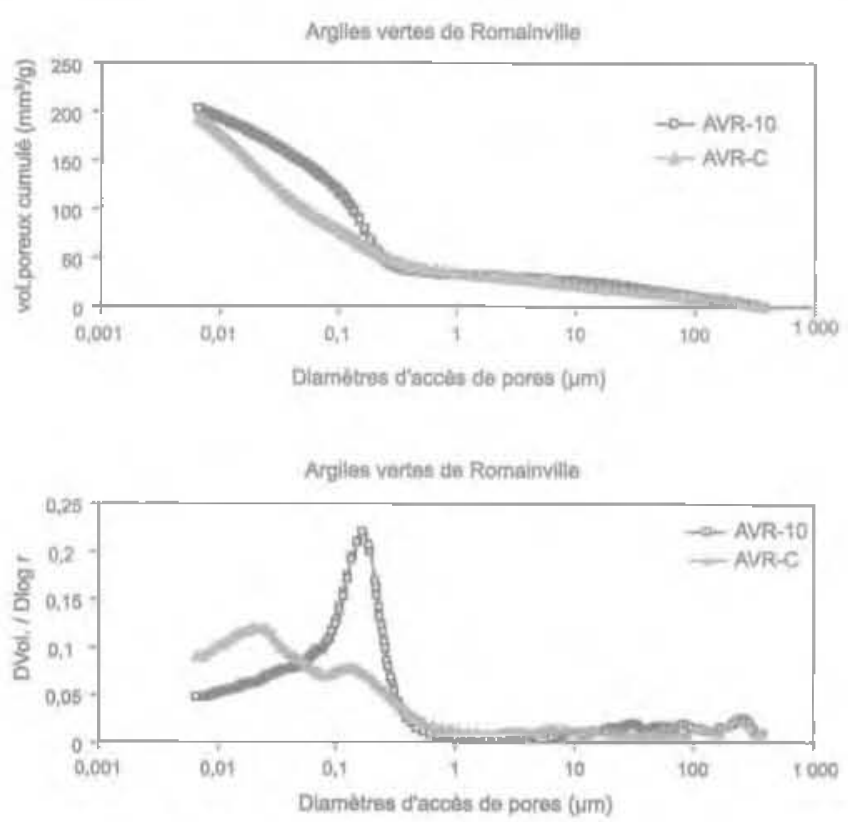

F19. 19 Courbes cumulées et dérivées de distribution des diamètres de pores de l'Argile verte de Romainville à l'état naturel (AVR-10) et à l'état de colluvion (AVR-C).

Cumulative and derived curves of pore size distribution of Argile verte de Romainville in natural state (AVR-10) and in colluvium state (AVR-G).
Sur les figures $13 a$ et b sont représentées respectivement les courbes porosimétriques cumulées et dérivées des trois états hydriques et sur les figures $14 \mathrm{a}, \mathrm{b}, \mathrm{c}$ les images correspondantes obtenues au MEB.

Le volume poreux, rapporté au poids sec, de l'échantillon séché à l'étuve est de $130 \mathrm{~mm}^{3} \mathrm{~g}^{-1}$ pour un diamètre moyen de $40 \mathrm{~mm}$. La microstructure correspondante est très compacte et le réseau poreux pratiquement inexistant ż l'échelie de l'observation au MEB. L'ensemble des amas de particules s'est rétracté l'échelle microscopique, ce phénomène se traduisant par l'apparition de lissures de rétraction à l'échelle macroscopique.

L'échantillon naturel lyophilisé présente un volume poreux rapporté au poids sec de lordre de $200 \mathrm{~mm}^{3} \mathrm{~g}^{-1}$ pour un diamètre moyen d'accès de pores de $150 \mathrm{~nm}$. L'observation au MEB met en évidence l'ouverture de microfissures de gonflement (porosité bidimensionnelle) qui initient dans la matrice argileuse la formation d'agrégats primaires ou qui révèlent une structure en agrégats déjà existante mais en partie oblitérée dans l'échantillon séché sous l'effet de la rétraction. Parallèlement à cela, les cristallites ou amas de particules commencent à se détacher les uns des autres.

Léchantillon gonflé librement a ur volume poreux rapporté au poids sec de $337 \mathrm{~mm}^{3} \mathrm{~g}^{1}$ pour une distribution des diamétres d"accès de pores présentant deux classes bien individualisées : une centrée sur $650 \mathrm{~nm}$, l'autre vers $55 \mathrm{~mm}$. Lanalyse au MEB met en évidence des microfissures de grande extension, d'épaisseur micrométrique, recoupées par d'autres plus petites
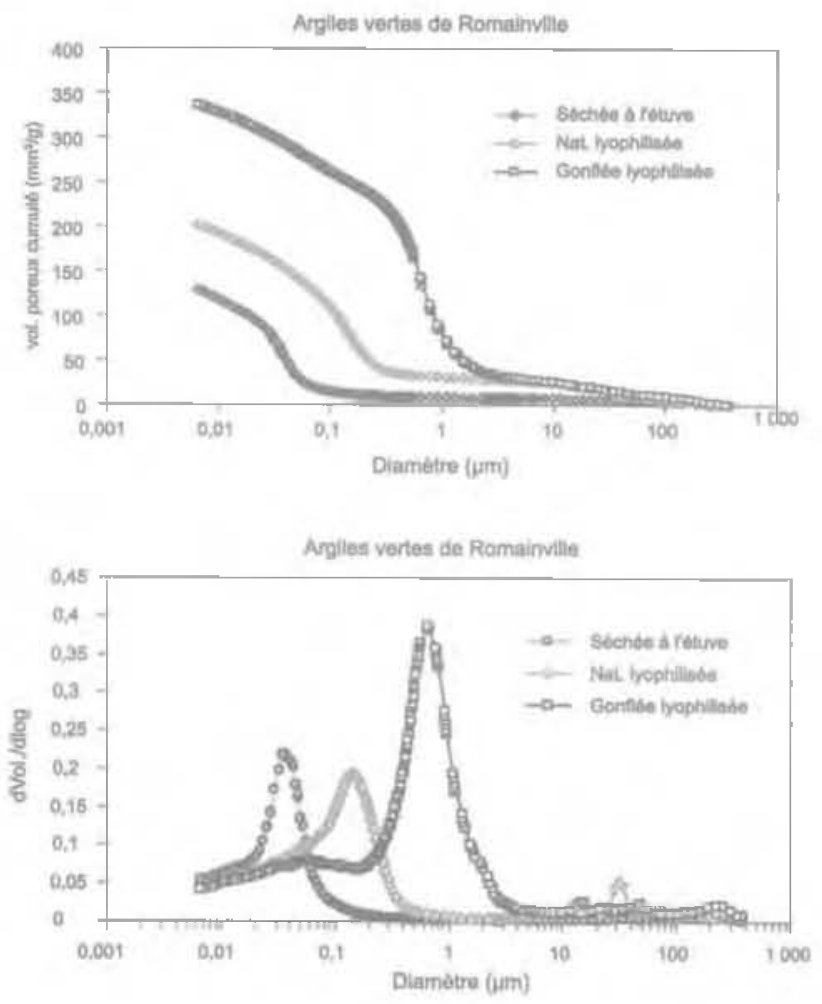

Flg. 13 Courbes cumulée et dérivée de distribution des pores de l'Argile verte de Romainville pour trois états d'hydratation.

Cumulative and derived curves of pore size distribution of Argile verte de Romainville for three hydration states. 


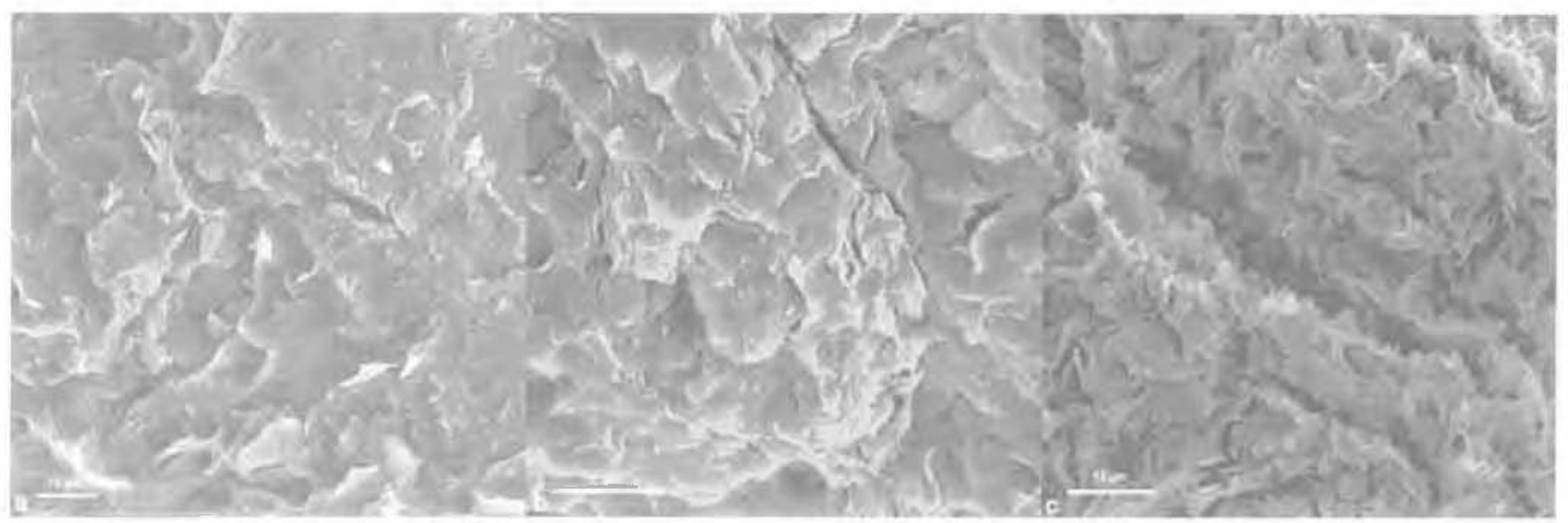

Fg, 14 Lmages au MEB de l'Argile verte de Homainville : a) état sec ; b) état naturel lyophilisé ; c) après gonflement libre et lyophilisation.

SEM micrograph of Argile verte de Romainvilie: a) dry state; b) Iyophilized natisal state; c] after free swelling and lyophilization.

gui subdivisent les agrégats. Les cristallites se détachent les uns des autres et les grains ce carbonates ou de quartz enrobés et disséminés dans la matrice argileuse sont mis a nus.

A partir de cette analyse les conclusions suivantes peuvent être établies

- au cours de l'hydratation la porosité augmente en taile et en volume par ouverture progressive et simultanée des porosités inter et intra-agrégat primaires, confondues à l'état dếshydratẻ, générant ainsi des agrégats secondaires :

- le processus de subdivision des agrégats se poursuit jusqu'à atteindre des amas de quelques particules, initant ainsi une famille de pores intra-agrégat ou interparticulaires distincte :

- la queue de distribution vers les faibles diamètres commune aux 3 états d'hydratation dénote l'existence d'une porosité intraparticulaire non accessible par la porosimétrie au mercure.

\section{4}

\section{Caractéristiques géotechniques}

Cette caractérisation repose, d'une part, sur les mesures des paramètres d'état (teneur en eau, poids volumiques humide et sec, porosité ou indice des vides) et des paramèzres de nalure (granularité, minéralogie) et, d'autre part, sur d'autres grandeurs qui rendent compte de lactivité du sol. En effet, la fraction argileuse < $2 \mu \mathrm{m}$ possède en présence d'eau des proprjétés de surface (cohésion, plasticité, retrait, gonflement) regroupées sous le terme a activité $n$. Ces propriétés résultent à la fois de la finesse des particules (argile au sens granulométriquel qui leur confère une grande surface spécifique externe, mais aussi de leur structure en feullets (argiles au sens minéralogique) qui leur permet de développer de grandes surfaces spécifiques externe et interne et qui présente un déficit de charge responsable des propriétés d'adsorption de cations et de molécules d'eau.

Trois principaux essais rendent compte de ces propriétés : les limites d'Atterberg, l'essai d'adsorption au bleu de méthylène, la mesure de capacité d'échange cationique.
Les limites d'Atterberg sont des teneurs en eau mesurées de façon conventionnelle qui séparent différents états du sol. La limite de liquidité w sépare l'état liquide de l'état plastique et est mesurée par l'essaj à la coupelle de Casagrande ou par la méthode du cône de pénétration (NF : P 94-051, P 94-052-1). La limite de plasticité $w_{p}$ sépare l'état plastique de l'état solide et est obtenue par la méthode du rouleau (NF : P 94051). Enfin, la limite de retrait w, sépare l'état solide avec retrait de l'état solide sans retrait sur échantillon remanté ou intact. Elle est obtenue par la construction graphique volume-teneur en eau schématisée sur la figure 15 et fait l'objet comme les essais précédents de deuX normes (XP P94-060-1 et XP P94-060-2).

A partir de ces trois grandeurs drautres paramètres sont définis

- l'indice de plasticité : $I_{p}=w_{1}-w_{p}$;

- l'indice de consistance : $I_{c}=\left(W_{L}-W_{n g}\right) / I_{n}$;

$-l^{\prime}$ indice de retrait : $I_{r}=W_{1}-w_{r}$;

- l'activité de Skempton: $\mathrm{A}=\mathrm{I}_{\mathrm{n}} /<2 \mu \mathrm{m}$.

Un sol aura une aptitude au gonflement d'autant plus importante que $w_{L^{\prime}} I_{p^{\prime}} I_{r^{\prime}}$ A seront grands et $w_{p}$ et $w_{r}$ petits. Le tableau II présente des valeurs de quelques paramètres concernant les familles d'argiles les plus courantes. Les smectites sodiques ont la plus grande aptitude au gonflement

L'essai d'adsorption de bleu de méthylène (NE P 94068) mesure la quantité de bleu nécessaire pour recouvrir d'une couche monomoléculaire par chimisorption, les surfaces internes et externes des particules présentant un deficit de charge (minéraux argileux et colloìdes essentiellement). Cette quantité de bleu adsorbée permet de mesurer la surface spécifique développée par les minéraux (Tran, 1977).

La capacité d'échange de cations est due aux effets de bords des feuillets et aux déficits de charge à l'intérieur de la structure provenant de substikutions d'ions de valence $n$ par des ions de valence n-1. Cette mesure peut être réalisée par des essais normalisés (NF X 31130) ou évaluée à partir d'un essai plus courant en géotechnique, l'essai au bleu de méthylène ( Cuisset, 1980; Laribi et al., 2007). 


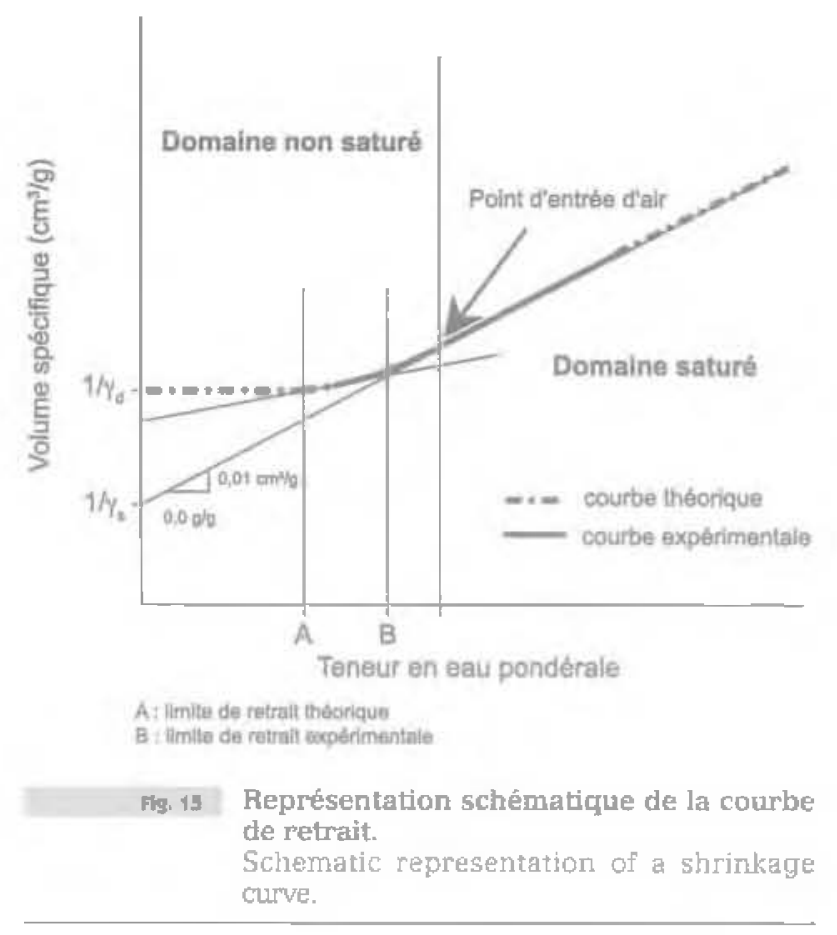

Des classifications permettant d'estimer le potentiel de gonflement d'un sol ont été élaborées en prenant appui sur ces différents paramètres. Elles ont fait l'objet de plusieurs synthèses notamment par Muntohar (2006) et Rao (2006). Les premières classifications concernant les sols gonflants ont été établies aux ÉtatsUnis dans les années 50. Elles tenaient comptent du pourcentage en particules collödales (d'après Holtz et Gibbs, 1956, dans Raa, 2006, tableau III). De nombreuses autres ont suivi, basées sur un ou plusieurs paramètres (quelques exemples sont donnés dans les tableaux IVà à $\mathrm{Vg}$ ), mais les résultats sont parfois discutables car le potentiel de gonflement d'un sol peut être qualifié de faible ou fort selon le paramètre considéré. Ainsi la classification de Holtz (dans Djedid, 2001) conduit à placer l'Argile verte de Romainville dans les sols argileux à fort potentiel de gonflement par référence aux valeurs de limite de liquiditê et d'indice de plasticité et à faible potentiel de gonflement par référence à la limite de retrait.

raveu II Valeurs des limites d'Atterberg d'argiles monominérales, (d'après Lambe et Whitman, 1969).

Walues of Atterberg limits of mono-mineral clayey sols, (after Lambe and Whitman, 1969).

\begin{tabular}{l|l|l|l|l|l} 
Minéral & $\begin{array}{l}\text { Cation } \\
\text { échangeahle }\end{array}$ & $\begin{array}{l}\text { Limite de } \\
\text { liquidité (\%) }\end{array}$ & $\begin{array}{l}\text { Limite de } \\
\text { plasticité (\%) }\end{array}$ & $\begin{array}{l}\text { Indice de } \\
\text { plasticité (\%) }\end{array}$ & $\begin{array}{l}\text { Limite de } \\
\text { retrait (\%) }\end{array}$ \\
\hline Montmorillonite & $\mathrm{Na}^{*}$ & 710 & 54 & 656 & 10 \\
\hline Mc & Ca & 510 & 81 & 429 & 11 \\
\hline TLite & & 120 & 60 & 60 & 18 \\
\hline Kaolinite & & 53 & 32 & 21 & 27 \\
\hline
\end{tabular}

wark III Classification géotechnique des sols gonflants (d'après Holtz et Gibbs, 1956). Geotechical classification of expansive soils (afterHoltz and Cilbos, 1956).

\begin{tabular}{|c|c|c|c|c|}
\hline $\begin{array}{l}\text { Colloidtes } \\
\text { [\%] }\end{array}$ & $\begin{array}{l}\text { Indice de } \\
\text { Plasticitế } I_{n}(\%)\end{array}$ & Limite de retrajt $\{\%\}$ & $\begin{array}{l}\text { Potentiel de } \\
\text { gonflement }\end{array}$ & $\begin{array}{l}\text { Gonflement estime } \\
\text { (variation de volume \%) }\end{array}$ \\
\hline $\begin{array}{l}<15 \\
13-23 \\
20-31 \\
>28\end{array}$ & $\begin{array}{l}<18 \\
15-28 \\
25-41 \\
>35\end{array}$ & $\begin{array}{l}<10 \\
10-20 \\
20-30 \\
>30\end{array}$ & $\begin{array}{l}\text { faible } \\
\text { moyen } \\
\text { élevé } \\
\text { très élewe }\end{array}$ & $\begin{array}{l}<10 \\
10-20 \\
20-30 \\
>30\end{array}$ \\
\hline
\end{tabular}

TALEaU Na, To Potentiel de gonflement établi à partir de la limitedeliquidité (d'après a) Dakshanamurthy et Faman, 1973 ; b) Chen 1975).

Liquid Limit as an indicator of swelling potontial (after a) Dakshanamurthy and Farnan, 1973; b) Chen 1975 .

\begin{tabular}{l|l}
$w_{1}[\%]$ & Classification \\
\hline 0 à 20 & non gonflant \\
\hline 20 à 35 & gonflement faible \\
\hline 35 à 50 & gonflement moyen \\
\hline 50 à 70 & gonflement êlevé \\
\hline 70 à 90 & gonflement très élevé \\
\hline 390 & gonflement critique \\
\hline &
\end{tabular}

\begin{tabular}{l|l}
$w_{1}(\%)$ & Potentiel de gonflement \\
\hline$>60$ & très élevé \\
\hline 40 à 60 & èlevé \\
\hline 30 à 40 & moyen \\
\hline$<30$ & faible \\
\hline
\end{tabular}


ZABLEW $\mathrm{wc}_{c}$ Sensibilité d'une argile au retrait-gonflement déterminée à partir de l'indice de plasticité (d'apres Prian et al, 2000

Plasticity index as an indicator of clay sensitivity to shrinkage-swelling (after Prian et al., 2000.

\begin{tabular}{l|l}
$\Psi_{\text {fri }}(\%)$ & Sensibilté \\
\hline$<12$ & Faible \\
\hline 12 à 25 & Moyenne \\
\hline $25 \mathrm{a} 40$ & Forte \\
\hline$\geq 40$ & Très forte \\
\hline
\end{tabular}

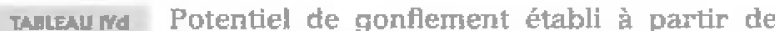
I'indice de retrait (d'après Ranganathan et Satyanarayana, 1965).

Shrinkage index as an indicator of swelling potential (after Fanganathan and Satyanarayana. 1965].

\begin{tabular}{l|l}
$I_{r}[\%]$ & Potentiel de gonflement \\
0 a 20 & Faible \\
\hline 20 a 30 & Moyen \\
\hline 30 a 60 & Fort \\
\hline 360 & Tres fort \\
\hline
\end{tabular}

tanreau ve Sensibilité d'une argile au retrait-gonflement déterminée à partír de la valeur de bleu (d'après Chassagneux et al. 1996).

Methylene blue value as an indicator of clay sensitivity to shrinkage-swelling (after Chassagneux et al, 1996).

\begin{tabular}{l|l} 
VBS & Sensibilité \\
\hline$<2,5$ & Faible \\
\hline 2,5 à 6 & Moyenne \\
\hline 6 à 8 & Forte \\
\hline$>8$ & Trè forte \\
\hline
\end{tabular}

uarcuumi Potentiel de gonflement (d'après Holtz et al. 1973).

Swelling potential (after Holtz et al', 1973)

\begin{tabular}{l|l|l|l}
$W_{p}(\%)$ & $W_{1}(\%)$ & $I_{y}(\%)$ & Potentiel de gontlement \\
\hline 7 à 12 & 50 à 70 & 25 a 35 & fort \\
\hline 10 à 15 & 35 à 50 & 15 à 25 & moyen \\
\hline$>15$ & 20 a 35 & $<18$ & falble \\
\hline
\end{tabular}

IAFEAU Ng Potentiel de gonflement (d'après BRE, 1980). Swelling potential (after BRE. 1980).

\begin{tabular}{|c|c|c|}
\hline$I_{p}(\%)$ & $\%<2 \mu m$ & Potentiel de goribanm! \\
\hline$>35$ & $>95$ & très élevé \\
\hline $22 \mathrm{a} 35$ & 60 à 95 & élevé \\
\hline 18 à 22 & 30 a 60 & moyen \\
\hline$<18$ & $<30$ & fatble \\
\hline
\end{tabular}

Il existe aussi des chartes de potentiel de gonflement : un exemple est celle établie nar Daksanamurthy et al. (1973) qui s'appuie sur le diagramme de Casagrande (Fig. 16).

On peut noter que ces classifications ne font pas souvent référence à l'état initial du sol.

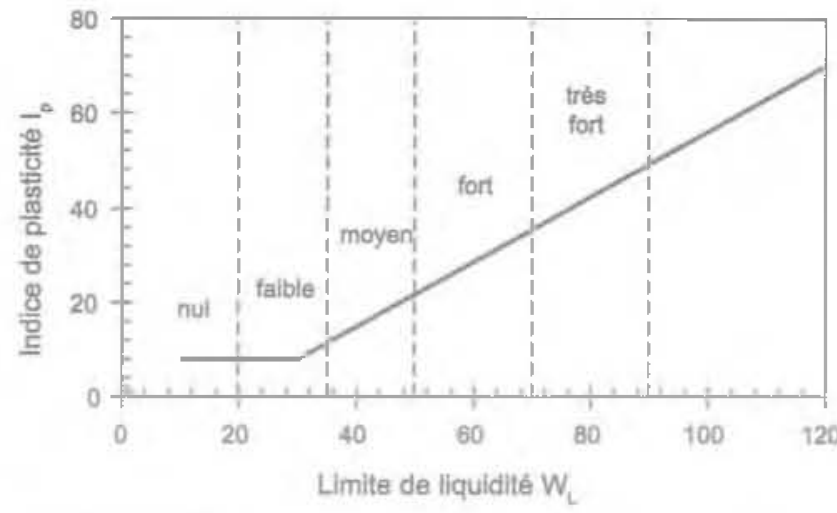

Fis. 16 Charte du potentiel de gonflement des sols (d'après Daksanamurthy et a', 1973). Chart of swelling potential of soil (after Daksanamurthy et al., 1973).

\section{Caractéristiques hydriques}

Les courbes de rétention d'eau sont établies au laboratoire en soumettant le sol à des contraintes hydriques ou succions variables. La succion appliquée à un sol est la différence de pression entre l'air et l'eau $\left(s=u_{a}-u_{\omega}\right)$. Elle correspond à une énergie potentielle, somme de plusjeurs termes: potentiels gravitaire, matriciel et osmotique. Elie s'exprime en unité die pression ou en $\mathrm{pF}$ (principalement chez les agronomes), le pF étant le logarithme décimal de la hauteur d'eau exprimée en centimètres, correspondant à la pression interstitielle dans le sol.

Les courbes de rétention traduisent la relation entre la succion et la teneur en eau ou l'indice des vides. Ces courbes présentent un phénomène d'hystérésis pouz les faibles succions correspondant à des teneurs en eau supérieures à la limite de retrait, Il n'existe donc pas de relation biunivocue entre la succion et la teneur en eau du sol, létat hydrique étant susceptible de varier entre deux courbes enveloppes d'imbibition et de drainage.

L'obtention de ces courbes de rétention nécessite différentes méthodes qui dépendent de la gamme de Succion appliquée (Delage et Cui, 2000) :

- les méthodes par plaque tensiométrique $(0-100 \mathrm{kPa})$ et par translation d'axes (jusqu'à 1,5 MPa);

- la méthode des pressions osmotiques (jusqu'á $12 \mathrm{MPa}$ )

- la méthode des solutions salines saturées qui permet d'atteindre des succions de l'ordre de $330 \mathrm{MPa}$. Elle offre l'avantage de couvrir une large gamme de succion, cependant elle présente l'inconvénient d'avoir des temps d'équilibre très grands dus aux transferts d'eau en phase vapeur

Les courbes de rétention d’eau peuvent être représentées de façon globale (Biarez et al. 1987) sous la forme de cing diagrammes, un exemple obtenu sur l'Argile verte de Romainvilie par la méthode des pressions osmotiques et la méthode des solutions salines saturées couplée à la mesure du volume par pesée hydrostalique dans le mercure, est dunné en figure 17. Sur le graphe a est représenté l'indice des vides en fonction de la teneur en eau permettant de déterminer la limite de retrait ( $w \%=14 \%, e_{0}=0,4$ ) correspondant à une succion de $20 \mathrm{MPa}$ (graphe b). Le matériau est 
saturé jusqưau point d'entrée d'air correspondant à une teneur en eau de $17 \%$ (graphe c) pour une succion de $4 \mathrm{MPa}$ (graphe d). Le processus de séchage-humidification est réversible pour des succions supérieures à la limite de retrait (graphe e).
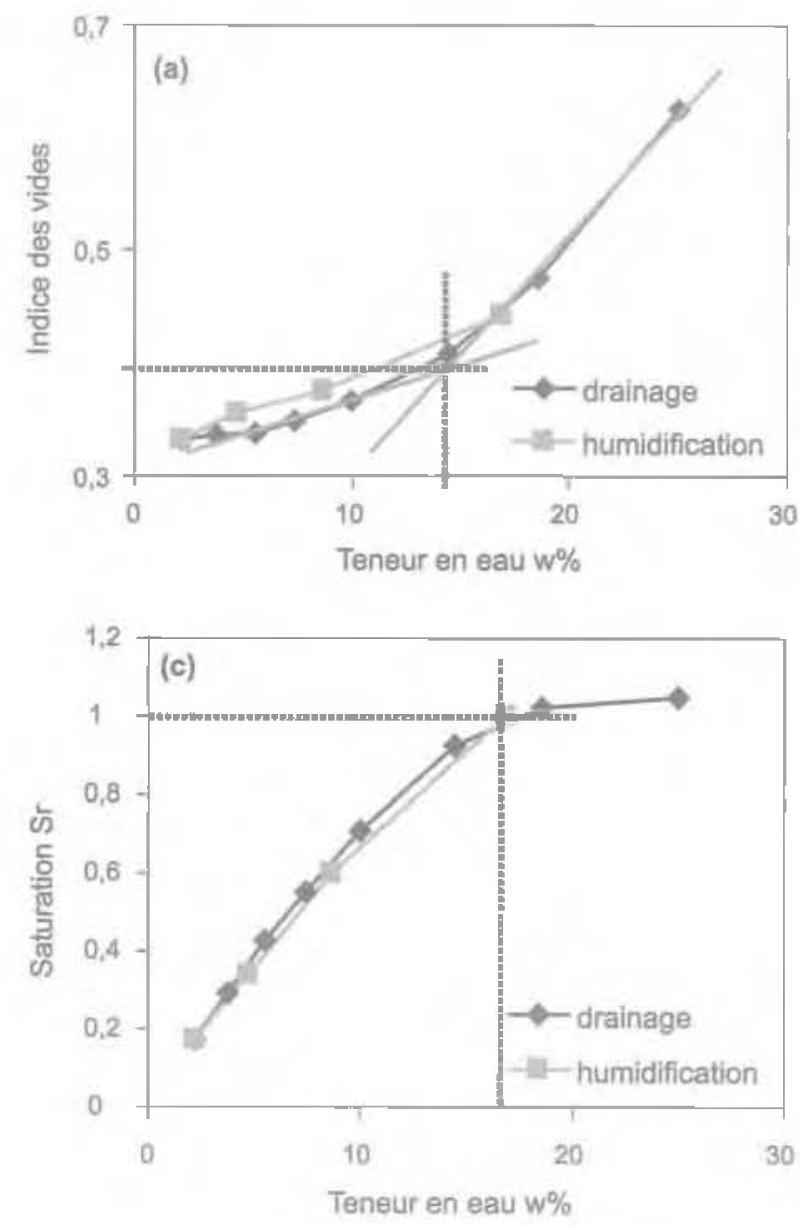

\section{Caractéristiques hydro-mécaniques}

Cet aspect est abordé pour l'Argile verte de Romainville à travers des essais cycliques de gonflement liłbre à l'oedomètre dans lesquels la variation de hauteur de
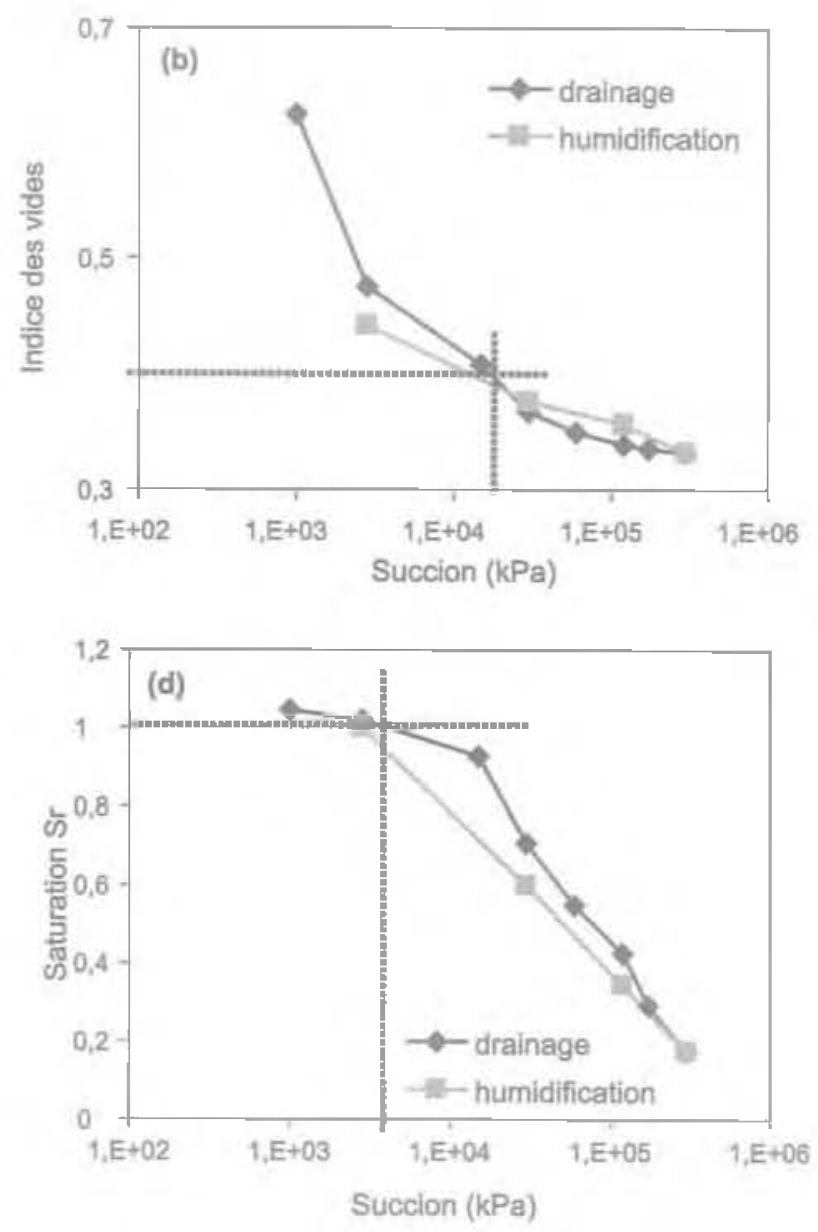

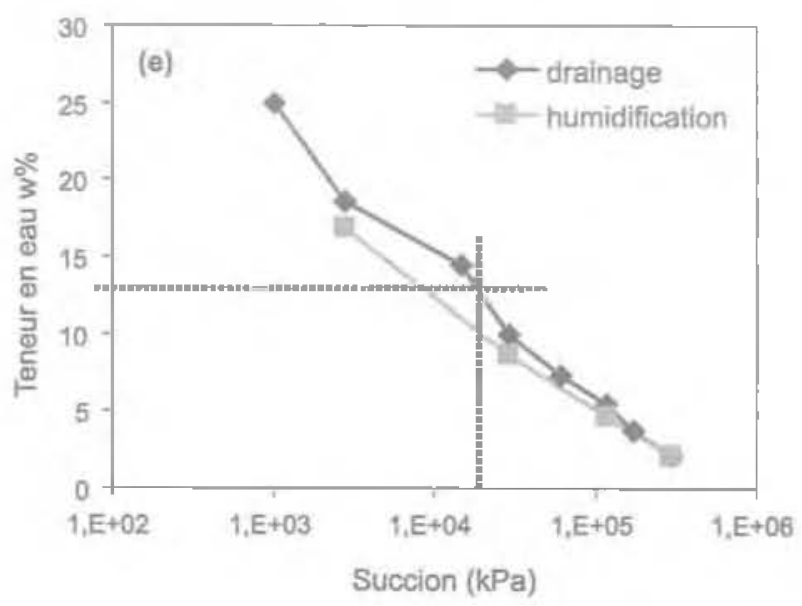

119. 1Y-1F Représentation globale des courbes de rétention de l'Argile verte de Romainville. Synthetic representation of retention curves of Argile verte de Romainville. 
l'échantillon est mesurée aw cours de cycles successifs séchage-humidification. En parallèle, une étude structurale par porosimétrie au mercure est réalisée.

Le chemin hydrique sujvi au cours des différents cycles est le suivant: l'échantilion mis en place dans le moule à l'état naturel (teneur en eaı et indice des vides naturels) est soumis à une phase d'humidification jusqu'à ce que le gonflement soit quasi stabilise, puis il est séché dans une êtuve à $50^{\circ} \mathrm{C}$ jusqu'à stabilisation des déformations. L'opération humidification-séchage est ensuite reproduite plusieurs fois et les courbes de gonflement sont analysées.

Sur la figure 18 sont représentées les courbes de gonflement obtenues sur un mème échantillon au cours de cycles successifs. La courbe de $1^{\text {er }}$ gonflement se détache des suivantes. Son allure et son amplitude sont influencées par l'état initial du matériau. La $2^{\circ}$ courbe présente une cinétique et une amplitude plus importantes quí peuvent être attribuées à la fissuration observée par ailleurs, au cours du 1'er séchage. En effet l'ouverture de físsures de retrait au cours du séchage entraine la création de chemins préférentiels empruntés par l'eau lors du processus de réhumidification suivant. Ce phénomène s'estompe ensuite pour les cycles suivants comme en témoigne la superposition des courbes de gonflement libre. La déformation se stabilise à partir du $3^{e}$ cycle (Fig. 19). Néanmoins l"échantillon ne retrouve pas son état initial à la din de chaque séchage, le phénomène n’étant pas entièrement réversible (Fig. 20j).

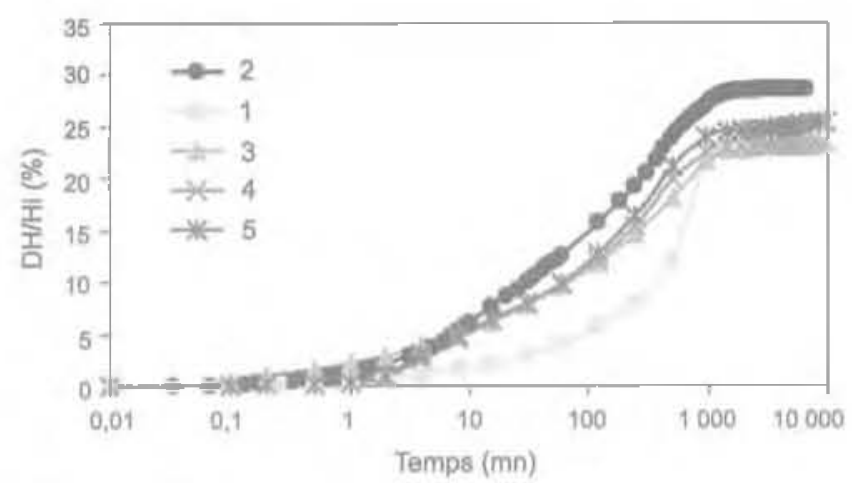

Fg. 19 Courbes de 5 cycles de gonflement libre à l'oedométre (Arctile verte de Romainville). Free swelling cedometer curves of 5 cycles (Argile verte de Romairnille\}

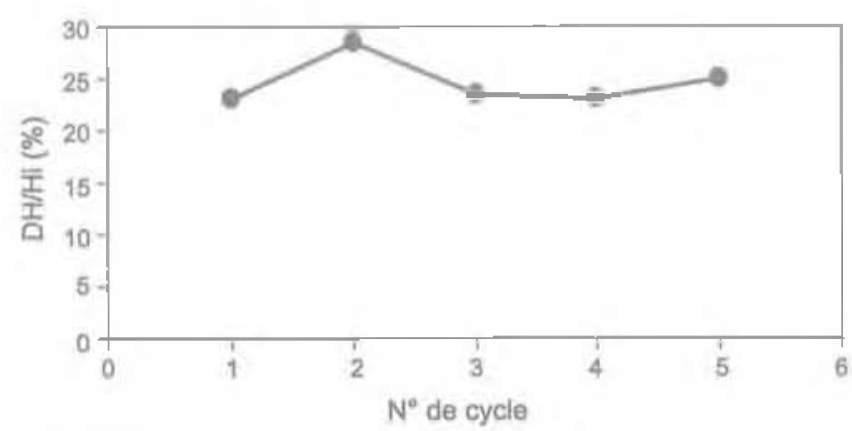

Fig. 19 Taux de gonflement au cours des 5 cycles (Argile verte de Romainville).

Sweling rate during the five cycles (Argite verte de Romainville].

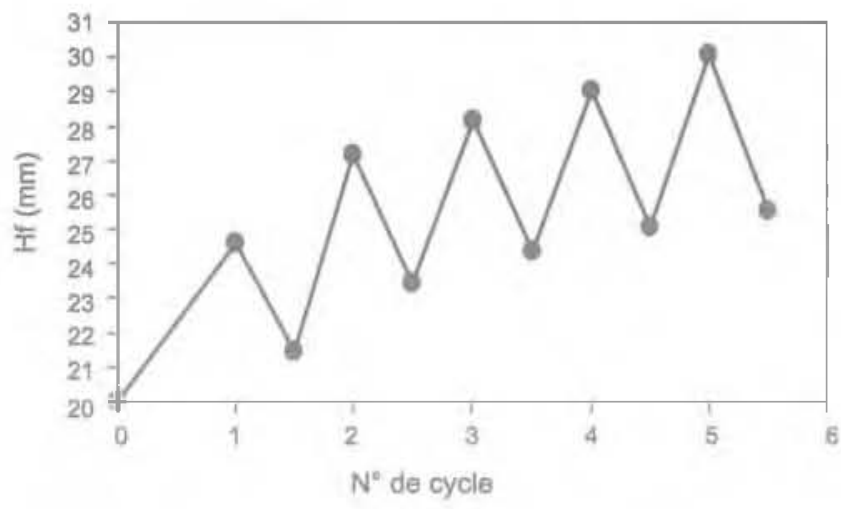

जg- so Évolution de l'épaisseur de l'échantillon au cours des 5 cycles (Argile verte de Romainville).

Thickness evolution of sample during the five cycles (Argile verte de Romainville).

L'évolution structurale qui résulte de ces différentes sollicitations hydriques est mise en évidence par I'étude porosimétrique. Sur les figures 21 a et 21 bont représentées les courbes cumulées et dérivées de la distribution des diamètres d'accès de pores d'échantillons ayant subi 1,3 et 5 cycles. On observe une augmentation du volume poreux cumulé entre le $1^{\text {er }}$ et le $3^{\text {ent }}$ cycle et une stabilisation entre le $3^{\mathrm{a}}$ et le $5^{\mathrm{e}}$ cycle. Ce résultat corrobore celui obtenu par les essais de gonflement libre où le taux de gonflement est constant à partir du $3^{e}$ cycle. Cependant, une modification de la microstructure est observée au cours des différents cycles, se traduisant par:

- une augmentation progressive du diamètre moyen de la famille de pores interagrégat;

- une distribution de plus en plus étalée du cycle 1 au cycle 5:

- une augmentation de la porosité intraparticulaire du cycle 3 au cycle 5. En effet la comparaison du volume poreux mesuré et de la teneur en eau correspondant aux trois cycles mentionnée sur le graphe des courbes cumulées, dénote pour le cycle 5 une part plus importante de la porosité non accessible par l"essai porosimétrique $(<7 \mathrm{~nm})$. Elle correspond à un volume poreux par unité de poids de l'ordre de $50 \mathrm{~mm}^{3} \mathrm{~g}^{-1}$ pour les cycles 1 et 3 et de $90 \mathrm{~mm}^{3} \mathrm{~g}^{1}$ pour le cycle 5 .

Ces essais hydro-mécaniques mettent en évidence que le matériau soumis à des cycles séchage-humidification ne présente pas d'évolution sensible du taux de gonflement au-delà des 2 ou 3 premiers cycles mais qu'une transformation progressive et irréversible de la microstructure est observable en porosimétrie. Cette évolution microstructurale se traduit à la fois par une augmentation des diamètres d'accès de pores de la famille interagrégat, une distribution plus étalée, et une augmentation de la porosité intraparticulaire.

7

\section{Conclusion}

Le travail qui fait l'objet de cet article, prend appuł pour les applications, principalement sur les Argiles vertes de Romainville et secondairement sur les Marnes bleues d'Argenteuil. Il permet, d'une part, de tirer des remarques générales concernant les facteurs de prédisposition au retrait-gonflement des sols argileux, 

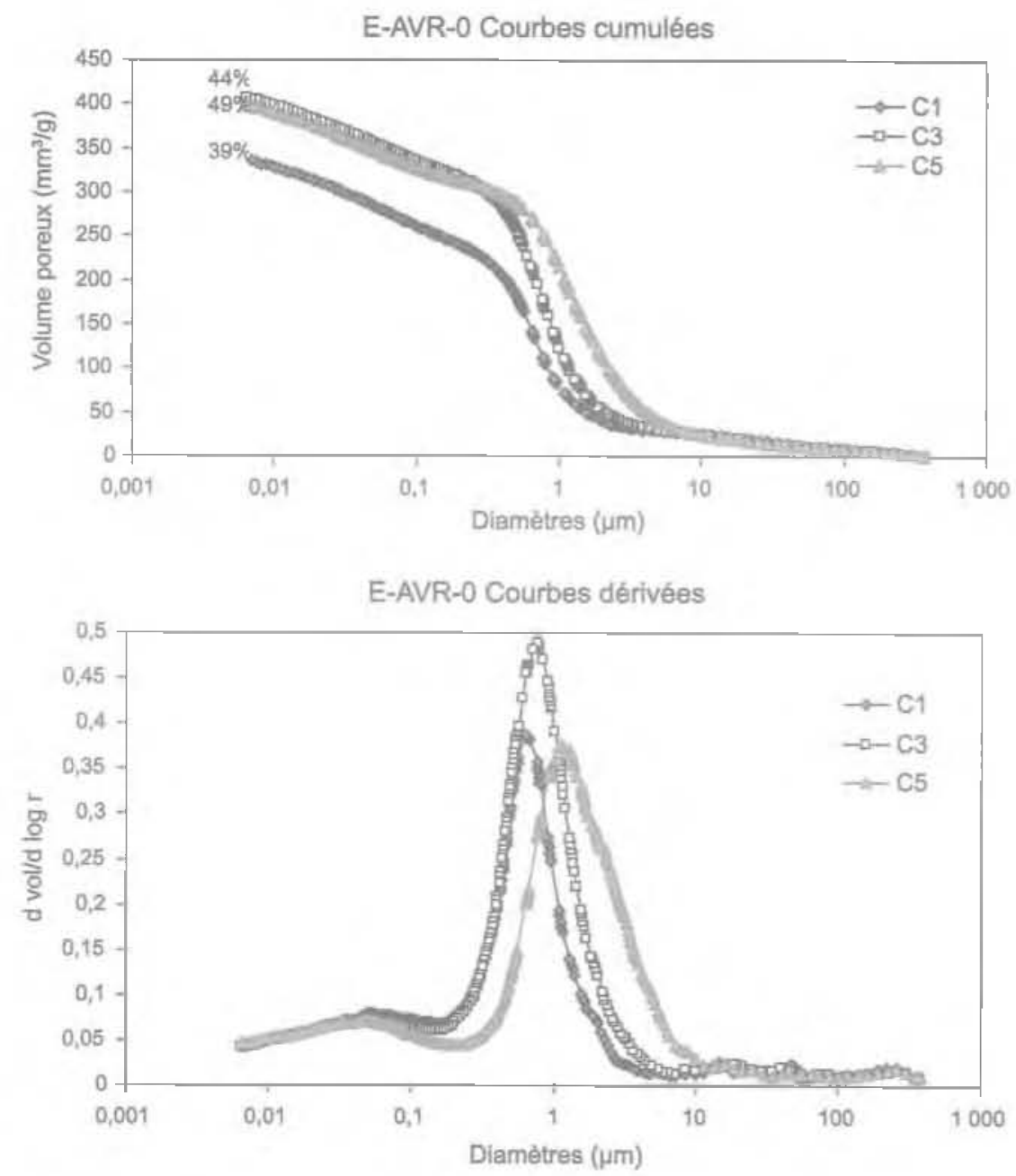

Fig. 2io ef 2ib Courbes cumulée et dérivée de distribution des pores de l'Argile verte de Romainville, pour les cycles $1,2,3$,

Cunulative and derived curves of pore size distribution of Argile verte de Romainville for cycles $1,2,3$.

et, d'autre part, d'étudier de manière plus spécifíque les modifications structurales de ce matériau lorsqu'il est soumis à des sollicitations hydriques. Les conclusions énoncées daris ce paragraphe sont structurêes suivant ces deux axes.

Les facteurs de prédisposition au retrait-gonflement sont nombreux et interdépendants. Il est impossible de les classer par ordre d’importance, chacun jouant un rôle déterminant à des échelles différentes. La liste qui suit, non exhaustive, correspond atux principaux critères permettant d'identifier les matériaux présentant des prédispositions aux variations de volume au cours de cycles séchage-humidification.

19) La nature minéralogłoue du sol :

- la présence de minéraux argileux et plus particulièrement de smectites et d'interstratifiés illite-smectite caractérisés par un déficit de charge et de très grandes surfaces spécifiques internes et externes responsables du phénomène d'adsorption de molécules d'eatl :

- la présence de carbonates qui jouent un rôle modérateur "le taux de gonflement libre à l'état natural de AVR est de 15 à $25 \%$ pour 15 à $20 \%$ de carbonates; celui de MBA est inférieur à $4 \%$ pour 30 a $60 \%$ de carbonates). Ils contribuent à former un squelette rigide freinant le processus de retrait-gonflement. Cependant ce squelette est très iragile et les liaisons sont facilement détruites par remaniement du matérlau (le taux de gonflement libre de MBA après remaniement est de $26 \%$ ), qui réagit alors en fonction des minéraux argileux présents dans la structure;

- la présence d'autres minéraux accessoires, sous forme d’amas ou lentilles, tels que la pyrite qui a été observée dans l’une des formations étudiées (Marnes bleues d'Argenteuil). Sous l'action de l'eau, la pyrite (FeS $S_{2}$ s'oxyde pour donner des sulfates qui en présence de calcium forment de l'anhydrite ( $\mathrm{CaSO}_{4}$ ) et par hydratation du gypse (CaSO, $2 \mathrm{H}_{2} \mathrm{O}$ ). Ce processus bien connu des géotechniciens est à l'origine de désordres par gonflement;

$2^{\circ}$ I'état initial du matériau. Il est mesuré par les paramètres d'état que sont la teneur en eau, la densité, l'indice des vides et la succion. A minéralogie et granularité égales, plus la teneur en eau initiale est faible, ou l"état de succion grand, plus le matériau est susceptible de s'bydrater, entraînant ainsi un gonflement dont l'amplitude dépend de la nature des minéraux présents et de l'arrangement structural. 
$3^{\circ}$ ) L'arrangement structural directement lié à la granularité et à la nature minéralogique du matériau. Plus la structure est compacte avec une distribution d'accès de pores unimodale correspondant à une matrice argileuse très resserrée, plus la capacité à se diviser en agrégats primaires ef secondaires au cours de I'hydratation est grande et plus les variations de volume observées sont importantes. Au contraire, une structure formée par une matrice granulaire ou par des agrégats où les minéraux non argileux sont dominants est moins sujette à des variations de volume.

4') Un paramètre important, cvoqué en introduction est l'état de consolidation directement ljé à l'histoire géologique du sol (processus de diagenèse et évolution ultérieure). Plus un matériau est surconsolidé, plus i] est susceptible de développer des pressions de gonflement importantes.

La plupart de ces facteurs de prédisposition sont identifiables et mesurables par des analyses minéra logiques, par des essais géotechniques simples et par l'étude de la microstructure.

Les nombreuses classifications géotechniques existantes permettent de placer les sols dans des catégories de sols au potentiel de gonflement nul à très fort. Cependant elles sont parfois discutables car basées sur seulement un, deux ou trois paramètres alors que le processus de retrait-gonflement est la conséquence de nombreux facteurs interdépendant's (granularité, minéralogie, état initial, microstructure...).

L'étude microstructurale de l'Argile verte de Romainville met en évidence :
- une augmentation de la porosité en taille et en volume au cours de l'hydratation du matériau naturel par ouverture progressive de la porosité inter et intraagrégat primaire ;

- une destructuration du matériau lorsqu'il se présente sous forme de colluvion se traduisant par la destruction des agrégats et favorisant l'apparition d'amas de quelques particules ou de particules dispersées;

- une transformation progressive et irréversible de la microstructure au cours des cycles successifs séchagehumidification, quí se traduit par une augmentation du diamètre d'accès de pores de la famille interagrégat et une distribution plus étalée sans variation sensible du volume poreux dont la conséquence directe, à l'échelle macroscopique, est l'absence d'évolution du taux de gonflement d’un cycle à l’autre au-delà du $3^{e}$ cycle.

Cette deniêre remarque est vérifiée pour 6 cycles mais reste à confirmer pour un plus grand nombre. La structure du matériau semble évoluer lentement vers une déstructuration qui à terme pourrait ressembler à celle des colluvions argileuses.

\section{REMERCIEMENTS}

Ces travaux ont été rêalisés dans le cadre du projet de recherche: "Aléa et risque sécheresse $\$$ souteñ par la Fondation MAIF, du projet ARGIC : A Analyse du retrait-gonflement et de ses incidences sur les constructions y soutenu par l'ANR et de la fiche recherche " Sécheresse géotechnique et bâtu u du Réseau de recherche sur le développement durable, soutenu par la région Ile-de-France.

\section{$\overline{\text { Bibliographie }}$}

AFNOA NF P 94-051 - Sols : reconnaissance et essais. Détermination des limites d'Atterberg. Limite de liquidité à la coupelle - Limite de plasticité au rouleau, 1993.

AFNOR NF P 94-052-1 - Sols : reconnaissance et essais. Détermination des limites d'Atterberg. Limlte de liquidité Méthode du cóne de pénétration, 1995.

AFNOR XP P 94-060-1 - Sols : reconnaissance et essais. Essai de dessication. Partie 1 : Passant 400pum, 1995.

AFNOR XP P 94060-2 - Sols ; reconnaissance et essais. Essai de dessication. Partie: Sol non remanié, 1995.

AFNOR NF P 94-068 - Sols : Reconnaissance el essais. Mesure de lá capacité d'adsorption de bleu de méthylène d'un sol ou d'un matéríau rocheux, 1998.

AFNOR NF X 31-130 Qualité des sols. Méthodes chimiques. Détermination de la capacité d'échange cationique (CEC) et des cations extractibles, 1999.

Al-Mukhtar M., Belanteur N., Tessier D., Vanapalli S.K. - The fabric of a clay soil under controlled mechanical and hydraulic stress states. Applied Clay Sciences, vol. 11, 1996, p.99-115.

Alonso E.E., Gens A, Josa A - A constitutive model for partially saturated soils. Geotechnique, vol. 40 (3), 1990, p. 405430.

Audiguier M. Delage P. 1987. Étude microscopique et porosimétrique de sols fins naturels dans une perspective géotechnique. Actes de la VIt Réunion internationale de micromorphologie des sols, Paris, ss la dir de N. Fedoroff, I.M. Bresson et M.A. Courty. juillet 1985 p. 493-500, AFES.

Biarez J., Fleureau J.-M., Zerhouni M. I., Soepandji B. S. - Variations de volume des sols argileux lors des cycles drainage-humidification. Revue française de géotechnique 41, $1987, p, 63-71$.

Chassagneux D., Stieljes L., Mouroux P., Ménilliet F. Ducreux G.H. - Cartographie de l'aléa retrait-gonflement des sols (sécheresse-pluie) à l'écheile départementale. Approche mêthodologique dans les Alpes-de-Haute-Provence. Rapport BRGM n ${ }^{\circ}$ R39218, 1996 .

Chen F.H. - Foundations on expansive soils. Amsterdam, Elsevier. Developments in Geotechnical Engineering, vol. 12 1975.

Collins $\mathrm{K}$, McGown A. - The form and function of microfabric features in a variety of raturals soils. Géotechnique, 24-2, 1974, p. 223-254.

Cuisset 0. - Propriétés électrocinétiques des particules argileuses, application de la méthode électrophonétique ân probiemes d'environnement et d'identification des sols. Rapport LCPC n ${ }^{\circ} 96$, 1980, P. 48-49.

Dakshanamurthy V., Raman V. - A simple method of identifying an expansive soil. Solls and foundations. Japanese Society of Soll Mechanics and Foundation Engineering, vol. 13, $\mathrm{n}^{\mathrm{n}} \mathrm{1}, 1973, \mathrm{~s} .97-104$.

Delage P. - Microstructure Features in the Behaviour of Engineered Barriers for Nuclear Waste Disposal. 5th Interna- tional Conference on Unsaturated Soils, Weimar, Germany, (à paraittre en 2007).

Delage P. Audiguier M., Cui Y. J., Howat M. - Microstructure of a compacted silty clay. Canadian Geotechnical Journal. vol. 33,1996, p. $150-158$

Delage P. Cuj Y. J. - L'eau dans jes sols non saturés. Techniques de l'Ingénieur, traité Construction. C 301, 2000, 20 p.

Delage P., Pellerin F.M. - Influence de la lyophilisation sur la structure d'une argile sensible du Québec. Clay Minerals 19,1984, p. 151-160.

Djedid A., Bekkouche A.. Ajssa Marnoune S.M. - Identification et prévision du gonflement de quelques sols de la région de Tlemcen (Algérie). Bulletin des $\triangle P C_{\text {. }}$ $\mathrm{n}^{\circ}$ 233, 2001, p. 67-75.

Durand C.. Forsans T., Ruffel C.. Onaisi A. Audibert A. - Influence of clay on borehole stability: a literature survey, Part one: Occurrence of drilling problems. physico-chemical description of clays and of their interaction with fluids. Rev. IFP, vol, 50, 1995, $\mathrm{n}^{\circ} 2$

Gens A. Alonso E E. - A tramework for the behaviour of unsaturated expansive clays. Canadian Geotechnical Journal, 29, 1992, p. 1013-1032

Hoitz W.G., Gibbs H.J. 1956. Engineering Properties of Expansive

Clays. Transactons, ASCE, vol. 121. 1956. p. $641-677$

Lambe T.W., Whitman R.V. - Soil Mechanics, Series in Soll Engineering. John Wiley \& Sons, Inc., New York, 1969. 
Laribi S., Cojean R., Audiguier M., Grambin-Lapeyre C., Geremew Z. - Influence de paramètres du protocole de l'essai d’adsorption at bleu de méthylène sur la valeur an bleu en fonction de la com. position minéralogique. Revue française de géotechnique $\mathrm{n}^{\text {os }}$ 120-121, numéro spécial sécheresse géotechnique, 2007.

Matyas E.L., Radhakrishna H.S. - Volume change characteristics of partially saturated sofls. Geotechnique, $18, n^{\circ} 4,1968$, p. $432-448$

Mitcheil J.K. - Fundamentals of Soils Behaviour. Series in Soil Engineering John Wiley \& Sons. Inc., New Yark, 1976.

Mitchell J.K. Soga K. - Fundamentals of Soils Behaviour. Johr Wiley, Mew York 2005.

Muntohar A. S. - Prediction and classification of expansive clay soils. Fecent advances in characterization and treat ment. Amer Ali Al-Rawas \& Mattheus F.A. (eds) Proceedings and Monographs in Engineering, Water and Earth Sciences, Goosen, Taylor \& Francis Group. Balkema, 2006.

Pomerol C., Feugueur L. - Bassin de Paris. lle-de-France, pays de Bray. Guides géologiques régionaux, Masson \& Cie. 1974.

Pons Ch., Rousseaux E., Tchoubar D. - Utilisation du rayonnement synchrotron en diffusion atux petits angles pour l'étude du gonflement des smectites. Clay Minera15 16, 1981, p. 23-42.
Prian J.-P., Donsimoni M., Vincent M. avec la collaboration de Denis L. Gallas J.C., Marty F. Motteau M. - Cartographie de l'aléa retrait-gonflement des argiles dans le département de l"Essonne. Rapport BRGM $n^{\circ}$ RP-50376-FR, 2000.

Prost R. - Relations eau-argile : structure et gonflement des matériaux argileux. Matérialix argileux : structure, propriétés et applications, sous la direction de A. Decarreau. Société française de minéralogie et de cristallographie, GFA, 1990.

Qi Yun - Comportement hydromécanique des argiles: couplage des propriétés micro-macroscopicues de la laponite et de l'hectorite. Thèse de doctorat, université d’Orléans, 1996.

Ranganathan et Satyanarayana - A rational method of predicting swelling poten tial for compacted expansive clays. Proceedings of the 6th international Conference on Solls Mechanics and Foundation Engineering, Montreal, "1955, p. 92-96.

Rao S. M. - Identification and classification of expansive soils. In expansive soils. Recent advarces in characterization and treatment. Amer Ali Al-Rawas \& Mattheus F.A. (eds) Proceedings and Monographs in Engineering. Water and Earth Sciences, Goosen, Taylor \& Francis Group, Balkema, 2006.

Robinet . I, C., Rahbaoki A., Plas F, Lebon P. 1996. A constitutive thermomechanical model for saturated clays. Engineering Geology, 41, 1996, p. 145-149

Saiyouri N., Hicher P. Y., Tessier D. -
Microstructural approach and transfer water modelling in highly compacted unsaturated swelling clays. Mechanics of cohesive-frictional materials, 5, 2000. p. 41-60.

Saiyouri N., Tessier D. Hicher P. Y. - Experimetal study of swelling in unsaturated compacted clays. Clays Minerals, 39 , 2004 . p. $469-479$

Tessier D. - Etude expérimentale de l'organisation des matériaux angileux: bydratation, gonflement et structuration au cours de la dessiccation et de la réhilmectation. Thèse de doctorat ès sciences, université Paris VIl 1984.

Touret $0_{\text {. }}$ Pons C. H., Tessier D., Tardy Y. - Etude de la répartition de l'eeus dans des argiles saturées Mg2+ aux fortes teneurs en eau. Clay Minerals 25, 1990, p. 217-233.

Tran N.L. - Un nouvel essai d'identification des sols : l'essai au bleu de méthylène. Bufletir de liaison des LPC, $\mathrm{n}^{\circ} 88,1977$. p. 136-137.

Turki - Hydratation des minerawx argileux gonflants. Theje universite Louis-Pasteur, Strasbourg, 1987.

Van Damme H. - L'eau et sa représentation. Mécanique des sols non saturés, sous la direction de $\mathrm{O}$. Coussy et J.-M Fleureau, Hermès Science publications, 2002, p.23-68.

Yong R.N. - Soil suction and soil-water potentials in swelling clays in engineered clay barriers. Engineering Geology, 54, 1999, p. 3-13 